\title{
A Full-Duplex MAC Tailored for 5G Wireless Networks
}

\author{
Lucas de Melo Guimarães $(\mathbb{D})$ and Jacir Luiz Bordim $(\mathbb{1})$ \\ Department of Computer Science, University of Brasília, 70910-900 Brasília, DF, Brazil \\ Correspondence should be addressed to Jacir Luiz Bordim; bordim@unb.br
}

Received 28 July 2017; Revised 1 November 2017; Accepted 17 January 2018; Published 18 March 2018

Academic Editor: Xianfu Lei

Copyright (C) 2018 Lucas de Melo Guimarães and Jacir Luiz Bordim. This is an open access article distributed under the Creative Commons Attribution License, which permits unrestricted use, distribution, and reproduction in any medium, provided the original work is properly cited.

\begin{abstract}
The increasing demands for high-data rate traffic stimulated the development of the fifth-generation (5G) mobile networks. The envisioned $5 \mathrm{G}$ network is expected to meet its challenge by devising means to further improve spectrum usage. Many alternatives to enhance spectrum usage are being researched, such as massive MIMO, operation in mmWave frequency, cognitive radio, and the employment of full-duplex antennas. Efficient utilization of the potential of any of these technologies faces a set of challenges related to medium access control (MAC) schemes. This work focuses on MAC schemes tailored for full-duplex antennas, since they are expected to play a major role in the foreseeable $5 \mathrm{G}$ networks. In this context, this paper presents a MAC layer technique to improve total transmission time when full-duplex antennas are employed. Several evaluations in different scenarios are conducted to assess the proposed MAC scheme. Numerical results show that the proposed scheme provides gains up to $156 \%$ when compared to a state-of-the-art full-duplex antenna MAC protocol. Compared to traditional half-duplex antenna MAC protocols, the proposed scheme yields gain up to $412 \%$.
\end{abstract}

\section{Introduction}

Existing and future services may have rigorous requirements for mobile network in terms of latency (round-trip time less than $10 \mathrm{~ms}$ ), throughput surpassing $5 \mathrm{~Gb} / \mathrm{s}$, and density up to $900 \mathrm{~Gb} / \mathrm{s} / \mathrm{km}^{2}$ [1]. To support these requirements and the increase of bandwidth-intensive multimedia services, the fifth-generation (5G) mobile wireless networks are being subject of intensive research in a wide number of areas [2]. Examples of services that may take advantage of the foreseeable $5 \mathrm{G}$ include Internet of things (IoT), vehicular networking, and mobile cloud computing [1].

Although 5G mobile wireless networks may provide several advantages, it also imposes a number of challenges to be overcome, including massive increase on the number of connecting devices, data volume, and real-time services [1]. To meet such challenges, 5G networks rely on the development of new schemes which are able to improve spectrum usage. In this regard, many approaches arise such as massive MIMO, cognitive radio, operation in mmWave frequency, and the employment of full-duplex antennas [24]. One should note that the adoption of any of these approaches in the $5 \mathrm{G}$ wireless networks scenario may require new medium access control (MAC) schemes in order to take advantage from its main characteristics. In this context, this work will focus on the challenges faced by MAC schemes designed to boost full-duplex communications, once fullduplex antennas are expected to play a major role in $5 \mathrm{G}$ mobile wireless networks $[2,5]$. Full-duplex antennas have many advantages over the half-duplex counterpart, such as higher throughput, lower end-to-end delay, lower congestion, and even better primary user detection in the context of cognitive radio [6]. However, full-duplex antennas are known to have higher packet loss ratio and lower link reliability and require a larger queue to process the receiving packets [6]. Furthermore, full-duplex antennas suffer from "selfinterference," which is the interference experienced by the full-duplex antenna when it is simultaneously transmitting and receiving $[2,5,6]$. In order to deal with this problem, many physical layer schemes were proposed, turning fullduplex antennas use to be feasible [5]. These developments fostered the research on MAC schemes tailored for fullduplex antennas. Zhang et al. proposed the FD-MAC (fullduplex MAC) which exploits the advantages of full-duplex 
antenna to transmit and receive concurrently. In the paper, the authors show how FD-MAC can be used to deal with different types of communication in a $5 \mathrm{G}$ mobile wireless network [5]. The FD-MAC is based on the IEEE 802.11 [7] channel reservation mechanism that requires the exchange of request to send (RTS) and full-duplex clear-to-send (FCTS) frames to avoid collisions and provide radio synchronization.

Full-duplex MAC protocols need to deal with some problems that are also present in half-duplex antennas, such as hidden and exposed terminal problems. In half-duplex antennas, the hidden terminal problem is commonly tackled with the aid of RTS/CTS frames. Although RTS/CTS prevents hidden terminal problem, it introduces significant latency to the overall transmission time as presented in [8]. Due to its criticality, channel reservation has been target of a number of works in the literature. In [9], the authors proposed a novel technique to reduce network latency during channel reservation where the nodes are employed with half-duplex, directional antennas. The proposed scheme, termed DPTCR, relies on pulse/tone signals to reduce network latency while performing channel reservation. Unfortunately, the above technique cannot be directly applied to $5 \mathrm{G}$ mobile wireless networks as the devices are expected to be empowered with full-duplex, omnidirectional antennas [9].

The main contribution of this work is to propose a new MAC technique, named Full-Duplex Tone Based MAC (FDT-MAC). FDT-MAC exploits the use of pulse/tone signals to improve channel reservation when the mobile stations are empowered with omnidirectional, full-duplex antennas. Several evaluations in different scenarios are conducted to assess the proposed MAC scheme. Numerical results show that FDT-MAC provides channel reservation in a fraction of the time spent with traditional schemes that rely on control frames. Our results show that FDT-MAC can provide throughput improvements surpassing 156\% when compared to FD-MAC [5]. When compared against a traditional halfduplex antenna MAC, the throughput gain surpasses $412 \%$. The above results show that FDT-MAC is able to properly exploit the advantages of full-duplex antennas.

The rest of this paper is organized as follows: Section 2 presents a brief review of the antenna model considered in this work. Next, in Section 3, an overview of existing schemes that deals with full-duplex antenna will be presented along with some considerations about its constraints. Section 4 presents the main contribution of this work. Section 5 shows the analytical results of several metrics used to compare FDT-MAC performance against traditional schemes. Finally, Section 6 concludes this work and points directions to further investigation.

\section{Antenna Model}

Some assumptions related to antenna model are presented next. In this work, the IEEE 802.11 standard [7] with regard to $\mathrm{MAC}$ protocol is referred to as $\mathrm{HD}-\mathrm{MAC}$, since it was tailored in the context of half-duplex antennas. As discussed in Section 1, self-interference occurs when an antenna is simultaneously transmitting and receiving frames. If ignored, self-interference may impair severely the performance of fullduplex communications. Hence, self-interference cancellation is target of many studies in the state of art, such as [5]. Our work terms the self-interference mitigation factor $K$ as a probability, $0 \leq K \leq 1$. So, $K$ denotes the probability of a communication be successful despite self-interference. That is, the higher $K$, the smaller the losses due to self-interference. As half-duplex antennas are not able to transmit and receive frames simultaneously, self-interference does not occur on half-duplex antennas. There are several techniques that almost cancel self-interference losses [10]. Therefore, a number of works related to full-duplex antennas consider the value of $K$ to be very close to 1 [5]. The impact of self-interference and $K$ will be further assessed in Section 5. Also, it is assumed that all frames are transmitted in omnidirectional mode. Furthermore, it is assumed that the antennas can transmit and receive pulse and tone signals. Pulse and tone signals are sinusoids that carry no information. The time consumed until an antenna detects a pulse/tone signal is at most $5 \mu \mathrm{s}[11,12]$. Usually, these signals are commonly employed and coupled with some other schemes to point a particular network condition [9]. Moreover, it is assumed that an antenna can detect the received signal strength and the angle of arrival of an incoming signal with a reasonable accuracy. Existing techniques can provide angle of arrival precision of $6^{\circ}$ for omnidirectional antennas [13]. If the omnidirectional antenna is considered as an array of directional antennas operating in omnidirectional mode, the referred precision can reach $1^{\circ}[14,15]$. Regarding received signal strength precision, it is reported to be up to $0.1 \mathrm{~dB}$ [16]. The propagation model is assumed to be the two-ray ground model which is commonly used in the related literature [17]. Ending the antenna model assumptions explanation, some important issues related to full-duplex antennas communication will be presented next.

\section{Related Work}

Some approaches of MAC protocols tailored for full-duplex antennas will be discussed. Regarding full-duplex wireless communications, it can be summarized in two types [5]:

(i) The three-node full-duplex wireless unidirectional communication, depicted in Figure 1(a) (Type 1).

(ii) The two-node full-duplex wireless bidirectional communication, depicted in Figure 1(b) (Type 2).

A MAC protocol tailored for full-duplex antennas is proposed by [5]. This protocol is termed full-duplex MAC (FD-MAC) and will be explained with the help of Figure 1. FD-MAC uses RTS/FCTS frames to avoid hidden terminal problem in a similar way to HD-MAC. To this end, the network allocation vector (NAV) proposed by IEEE 802.11 [7] is used to avoid collisions. FD-MAC relies on the information of RTS/FCTS frames to know when the channel is busy, thus avoiding collisions $[5,18]$. The main singularity of FD-MAC is the use of FCTS frame, which is slightly different from clear-to-send (CTS) frame used in HD-MAC, since FCTS frame has two destination fields and two duration 


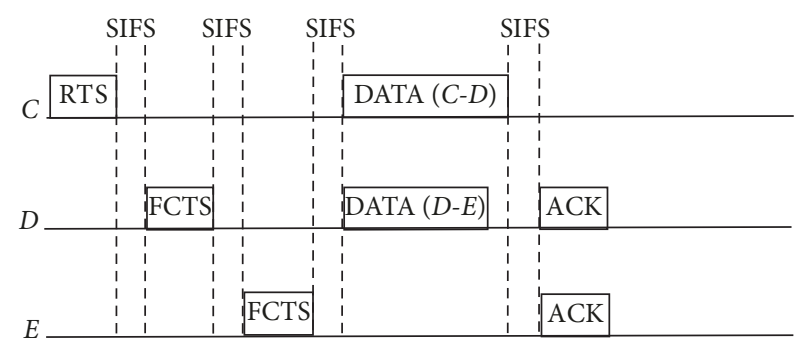

(a) Type 1: unidirectional links

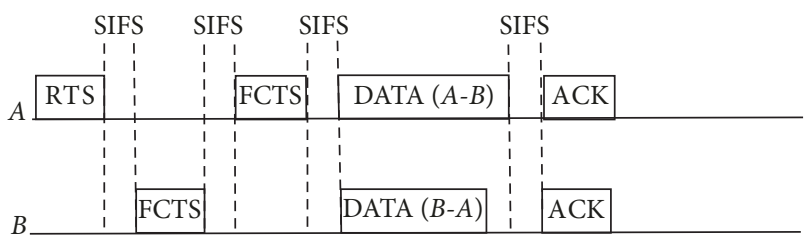

(b) Type 2: bidirectional links

Figure 1: Example of a communication using FD-MAC.

fields, instead of one. The two destination fields are needed to deal with communications of type 1 (Figure 1(a)), since in this case the FCTS sent from node $D$ simultaneously confirms the receipt of RTS from node $C$ and initiates a communication with node $E[5,18]$. The two duration fields are used to synchronization issues of the network, since the data packet size of the two ongoing transmissions may vary. For example, in Figure 1(b) the DATA packet that $A$ wants to send to $B$ can be larger than the one that $B$ wants to send to $A$. A similar argument is valid for communications of type 1 and also for different channel rates of the links. So, from these two durations of FCTS, the bigger one is chosen to guarantee the synchronization of the nodes, avoiding collisions $[5,18]$. Note that NAV uses this chosen time to allow a proper collision avoidance. Another difference to HD-MAC is that a communication can be initiated by a FCTS, as in communication of type 1 (Figure 1(a)) where $D$ initiates a communication with $E$ using a FCTS frame.

Although FD-MAC is able to provide a proper communication under full-duplex antennas, it has some drawbacks. Our work aims to overcome or at least mitigate these problems that are presented next. FD-MAC channel reservation has high latency, since there are more efficient channel reservation schemes than the one based on frames [9]. For instance, to transmit a packet of 256 bytes over a link of $11 \mathrm{Mbps}$, control frames exchange (RTS, FCTS, and ACK) constitutes more than $52 \%$ of the total time expended by FDMAC. Another issue unexplored by FD-MAC is related to the following scenario. Assume that $T_{\text {DATA }}^{i j}$ denotes the time spent to transmit the payload of the data incoming from node $i$ to $j$. Consider an ongoing communication as the one depicted in Figure 1(b). In this case, FD-MAC considers that the time spent with data packet $\left(T_{\text {DATA }}\right)$ is $\max \left(T_{\text {DATA }}^{A B}, T_{\text {DATA }}^{B A}\right)$. However, if $T_{\mathrm{DATA}}=T_{\mathrm{DATA}}^{A B}$, FCTS is not needed since its neighbors already overheard the RTS with the proper time of the whole communication. Also, it would be interesting to reduce FD-MAC time spent with acknowledgment (ACK) of data packets, thus providing gains in terms of network throughput. Furthermore, in some situations one of the data transmission times can be much higher than the other one which may not lead to a good usage of the network resources when compared against a policy that may fragment this larger packet in more than one. Note that this division would benefit the link of the shorter time since it would be available to start a new communication.
Therefore, our work proposes a technique which is able to deal with these issues, as it will be further discussed. Next section presents this technique which is our main contribution.

\section{MAC for Full-Duplex Communications}

This work proposes a tone based technique (FDT-MAC) for full-duplex antenna communications. FDT-MAC includes many of the main characteristics of FD-MAC presented in $[5,18]$, especially those related to physical layer. Furthermore, FDT-MAC is able to deal with some of the main problems of FD-MAC, providing gains in terms of network throughput. In order to achieve this performance gain, there are three main principles of FDT-MAC that differ from traditional schemes such as FD-MAC. The referred principles are the following:

(i) The RTS, FCTS, and ACK frames are replaced by pulse/tone signals.

(ii) In a bidirectional communication, node $A$ sends the tone to node $B$ if and only if it is necessary for NAV update. This behavior is referred to in this work as tone suppression.

(iii) In both bidirectional and three-node unidirectional communications, if there are two ongoing communications it would be interesting that none of the transmitters get idle waiting for the other ongoing communication ends. This property provides a better spectrum usage and may also increase total throughput along the time. This property is referred to as uniform data mode.

These principles aim to improve the network throughput by reducing the time wasted with channel reservation in full-duplex antenna communications. The first principle effectively reduces this time, since pulse/tone signals can be transmitted faster than frames (RTS/FCTS, ACK), as will be further discussed in Section 5. However, pulse/tone signals do not have MAC header, unlike frames. So, it is necessary to design a way to encode the main information contained in the MAC header (source, destination, and duration) into pulse/tone signal. This encoding is critical for FDT-MAC operation and will be further detailed. Both unidirectional and bidirectional examples of communication with FDTMAC are depicted in Figure 2. 


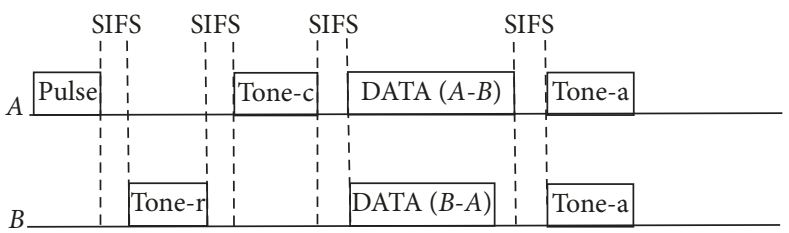

(a) Bidirectional links

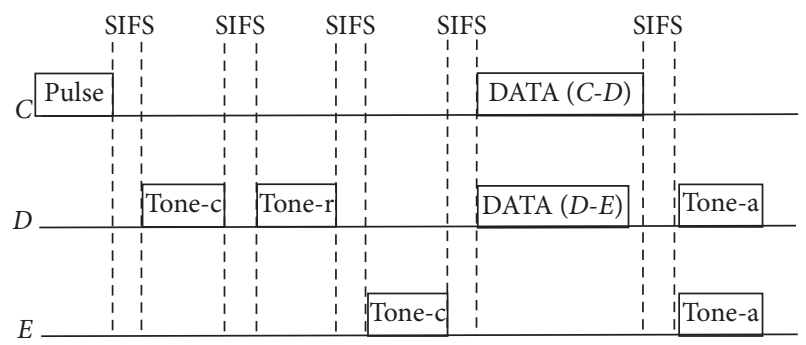

(b) Unidirectional links

FIgURE 2: Example of a communication using FDT-MAC.

Figure 2(a) shows an example of communication over a bidirectional link. In this case, it is assumed that node $A$ has data packets to send to $B$ and $B$ also has packets to send to $A$. First, node $A$ waits until its backoff counter reaches zero, in a similar way to that proposed in IEEE 802.11 standard. If the antenna is not blocked, node $A$ transmits a pulse to $B$. Node $B$ receives the incoming pulse and "detect" that it is bounded to it and that it came from $A$. So, node $B$ sends a tone request (tone-r) as response to $A$. As occurring in FD-MAC, the "field" duration of the tone-r signal is the maximum value between $\operatorname{DATA}(A, B)$ (encoded in the pulse) and the packet size that $B$ has to send to $A(\operatorname{DATA}(B, A))$. In an analogous way, node $A$ "confirms" that the received tone-r signal is bounded to it and that it has been sent by node $B$. Node $A$ replied to $B$ a tone confirm (tone-c) setting the field "duration" to the maximum value between $\operatorname{DATA}(A, B)$ and $\operatorname{DATA}(B, A)$. Neighboring nodes overhearing pulse, tone$\mathrm{r}$, and tone-c signals become aware of the communication between nodes $A$ and $B$ and set their NAV properly. Then, nodes $A$ and $B$ proceed to exchange DATA frames and tone-a signals instead of ACK frames. Figure 2(b) presents an example of communication over an unidirectional link where node $C$ has data packets to send to node $D$, while $D$ also has packets to send to node $E$. So, node $C$ transmits a pulse signal to $D$. At this time, node $D$ needs to reply to $C$ and also start a communication with $E$. However, a tone signal cannot be "detected" as bounded simultaneously to nodes $C$ and $E$ which forces node $D$ to send a tone-c to $C$ and then a tone-r to $E$. $C$ receives the tone-c and waits until $D$ establishes communication with $E$, while $E$ replies to $D$ a tone-c. Then, nodes $C, D$, and $E$ exchange DATA frames and tone-a signals. For the unidirectional links, the "duration" field of pulse, tone-r, and tone-c signals is built and likewise explained for bidirectional links using the maximum payload size of the communication. Again, NAV is properly updated by the overhearing nodes of the referred signals.

The second principle of FDT-MAC aims to avoid an unnecessary tone to be sent. Suppose that $\operatorname{DATA}(i, j)$ denotes the packet payload size from $i$ and is bounded to $j$. Therefore, if $\operatorname{DATA}(A, B) \geq \operatorname{DATA}(B, A)$, note that the tone-c sent from $A$ to $B$ confirming the size of the payload chosen as $\max (\operatorname{DATA}(A, B), \operatorname{DATA}(B, A))$ is unnecessary, since in this case $\max (\operatorname{DATA}(A, B), \operatorname{DATA}(B, A))=\operatorname{DATA}(A, B)$. Note that the $A$ neighbors had already updated its NAV properly, when they overheard the communication duration of the

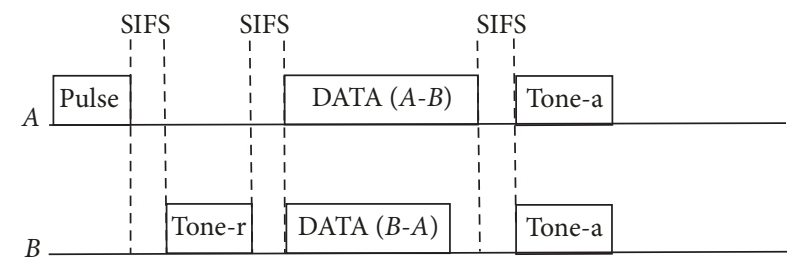

FIGURE 3: Example of tone suppression for FDT-MAC.

pulse sent from $A$. So, in this case the tone-c sent from $A$ to $B$ is suppressed. Hence, instead of transmitting tone-c, $A$ starts to send the DATA frames as displayed in Figure 3. Also, tone suppression saves one SIFS time of the total communication time, thus improving network throughput as will be showed in Section 5.

The third principle aims to enhance resource usage which is paramount in the context of resources sharing. When uniform data mode is enabled in FDT-MAC, the size of the payload is chosen as $\min (\operatorname{DATA}(A, B), \operatorname{DATA}(B, A))$, instead of $\max (\operatorname{DATA}(A, B), \operatorname{DATA}(B, A))$. Thus, uniform data mode assures that neither $A$ nor $B$ will be idle during the ongoing communication, since both will have the same payload size to transmit each other. The remaining of the payload $\max (\operatorname{DATA}(A, B), \operatorname{DATA}(B, A))$ is further transmitted in a new communication, while the node that already ended to send its payload is free to start a new communication to any node. Hence, the uniform data mode may improve network throughput depending on the conditions of the network traffic. An example of uniform data mode operation is presented in Figure 4. In this scenario, there are three payloads to be transmitted over a bidirectional link: two payloads of size 512 each from $A$ to $B$ and one payload of 1024 bytes from $B$ to $A$. When uniform data mode is enabled, the payload size is chosen as $\min (\operatorname{DATA}(A, B), \operatorname{DATA}(B, A))$ and the nodes do not get idle in the first communication, as depicted in Figure 4(a). In the second communication, the remaining data is transmitted along with the second payload from $A$ to $B$, thus allowing a higher resource usage and throughput than the traditional approach depicted in Figure 4(b). Further analysis over the advantages and limitations of data uniform mode are presented in Section 5.

4.1. Encoding Duration and Source/Destination. The encoding of MAC header main information into pulse/tone signals 


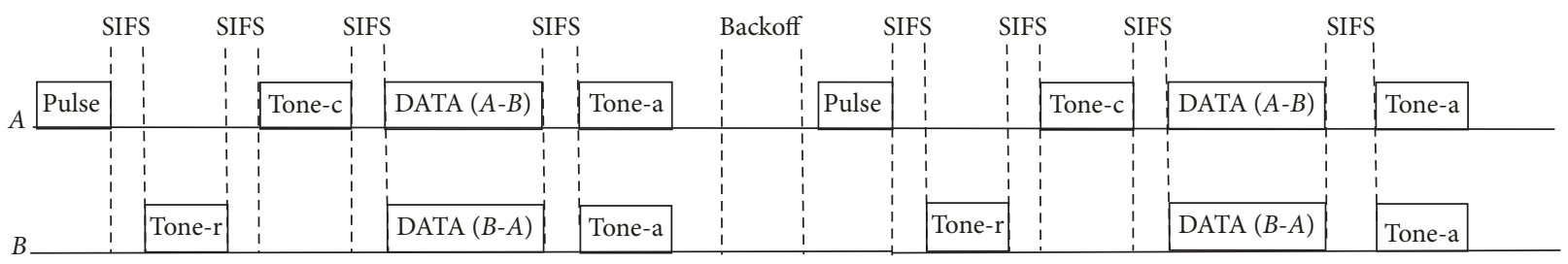

First communication

Second communication

(a) Uniform data mode

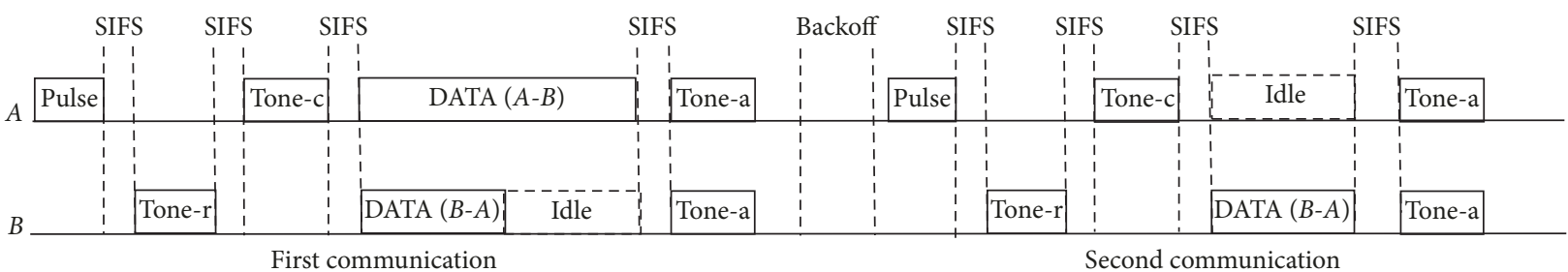

(b) Traditional approach

FIGURE 4: Comparison between uniform data mode and traditional approach of FDT-MAC.

plays an important role on FDT-MAC. There are three main pieces of information to encode: source of the signal, destination of the signal, and duration of the communication. The duration of the communication is encoded in the duration of the pulse/tone transmission $\left(T_{p}\right)$. So, for FDT-MAC, $T_{p}=$ $T_{\text {sync }}+\left\lceil\log _{2} P_{\mathrm{sz}}\right\rceil$, where $P_{\mathrm{sz}}$ is the data packet payload size (in bytes) and $T_{\text {sync }}$ is the necessary synchronization time. As discussed in Section 2, pulse/tone signals can be detected in at most $5 \mu \mathrm{s}$. Thus, it is considered that $T_{\text {sync }}$ equals $5 \mu \mathrm{s}$. Note that $T_{p}$ duration is the same for every signal used in FDTMAC (pulse, tone-r, tone-c, and tone-a). These signals can be differentiated by frequency, phase, or amplitude [17]. Therefore, when a node receives a signal, it can predict the duration of the communication from the duration of this signal. The source/destination of the signal is identified by searching in a lookup table for the combination of RSSI (Received Signal Strength Indicator) and AOA (Angle of Arrival). A node perceives that a signal is not bounded to it when the RSSI is higher than expected $[19,20]$. This lookup table could be mounted from the broadcast messages used by routing and MAC protocols, since these messages are periodically used in wireless communications [21]. These broadcast messages explicitly identify the source/destination nodes and allows every node to achieve RSSI/AOA information received from every neighbor, thus allowing the building of its own lookup table properly. As can be inferred, the encoding of source/ destination must not lead to wrong identification. In other words, this identification shall be very accurate to turn FDTMAC feasible. Next, a probabilistic analysis of the accuracy of that identification will be presented.

4.2. Identification Accuracy Analysis. Consider the notations presented in Notations section. An analysis about the accuracy of a similar mechanism designed for directional communications is addressed in [9]. Recall that FDT-MAC is tailored for omnidirectional communications. Hence, the aforementioned model cannot be directly applied. As will be explained next, we adapted the model in [9] to the FDT-MAC case.

Let node $x$ have at least two neighbors, say $y$ and $z$. The identification of source/destination fails if the following inequalities simultaneously occur:

$$
\begin{aligned}
& |R(x, z)-R(x, y)|<\tau ; \\
& |A(x, y)-A(x, z)|<\epsilon .
\end{aligned}
$$

So, considering that these events are independents, the analysis consists of the calculation of the following equation:

$$
\begin{aligned}
P_{f}= & P(|R(x, z)-R(x, y)|<\tau) \\
& \cdot P(|A(x, y)-A(x, z)|<\epsilon) .
\end{aligned}
$$

As explained in [9], the probability represented by these inequalities can be calculated using an analogy with the well-known Birthday problem (BP) [22]. The BP consists in computing the probability of at least two people having the same birthday. If the number of possible measures of RSSI was known as a function of $\tau$, this number could be interpreted as the number of days in a year, while $\tau$ would be one day of the year. Note that the possible measure of RSSI as a function of $\tau$ is $\left(R_{\max }-R_{\min }\right) / \tau$. Also, a similar argument can be used to AOA. However, AOA has an expected uniform distribution of probability, while RSSI does not [9]. In [22], equations are presented to solve both uniform and nonuniform cases of BP.

An example of what was discussed above will be presented with the aid of Figure 5. The figure depicts three different areas $\left(A_{1}, A_{2}\right.$, and $\left.A_{3}\right)$ of node $x$ antenna. According to the above expressions, prediction error occurs when two nodes (say $y$ and $z$ ) reside in the same area. In other words, node $x$ would not be able to correctly distinguish the incoming signal from either node $y$ or $z$, leading to problems in the communication. This would occur since these areas cover a circular sector of at most $\epsilon$ degrees and $|R(x, z)-R(x, y)|<\tau$.

To conclude the analysis of FDT-MAC source/destination identification accuracy, a numerical calculation of $P_{f}$ has 
TABLE 1: Parameters used for computing $P_{f}$.

\begin{tabular}{lcccc}
\hline Parameter & Transmitter & Receiver & Medium & Network \\
\hline pow & $15 \mathrm{dBm}$ & $\mathrm{N} / \mathrm{A}$ & $\mathrm{N} / \mathrm{A}$ & N/A \\
$g$ & $0 \mathrm{~dB}$ & $0 \mathrm{~dB}$ & $\mathrm{~N} / \mathrm{A}$ & N/A \\
lo & $1.4691 \mathrm{~dB}$ & $1.4691 \mathrm{~dB}$ & $\mathrm{~N} / \mathrm{A}$ & N/A \\
$h$ & $1.5 \mathrm{~m}$ & $1.5 \mathrm{~m}$ & $\mathrm{~N} / \mathrm{A}$ & N/A \\
$R_{\min }$ & $-94 \mathrm{dBm}$ & $-94 \mathrm{dBm}$ & $\mathrm{N} / \mathrm{A}$ \\
$R_{\max }$ & $0 \mathrm{dBm}$ & $0 \mathrm{dBm}$ & $4 \mathrm{~dB}$ & N/A \\
$\mathrm{sh}_{\operatorname{med}}$ & $\mathrm{N} / \mathrm{A}$ & $\mathrm{N} / \mathrm{A}$ & 0.125 & N/A \\
$w$ & $\mathrm{~N} / \mathrm{A}$ & $\mathrm{N} / \mathrm{A}$ & $\mathrm{N} / \mathrm{A}$ & N/A \\
$\Delta(G)$ & $\mathrm{N} / \mathrm{A}$ & $\mathrm{N} / \mathrm{A}$ & 5 \\
\hline
\end{tabular}

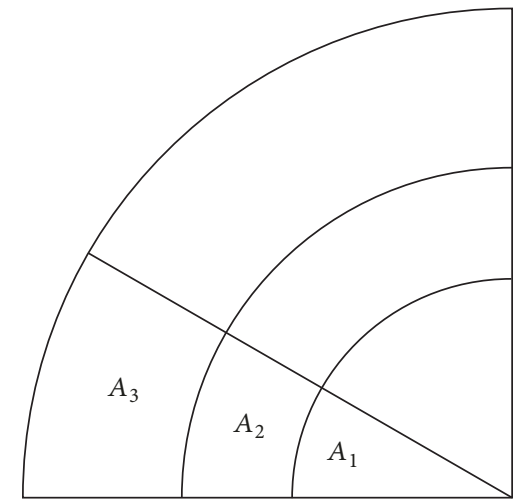

Figure 5: Example of areas in which the existence of two nodes may cause prediction error of the signal source.

been done. In regard to $\epsilon$, there are studies that claim the possibility of achieving a precision $\mathrm{AOA}$ of $6^{\circ}$ for $\mathrm{AOA}$ using omnidirectional antennas [13]. Moreover, RSSI precision can achieve even $0.1 \mathrm{~dB}$ as reported by [16]. Hence, assuming these values ( $\epsilon=6, \tau=0.1)$, as well as assuming that the network/antenna characteristics have the values displayed in Table $1, P_{f}$ is less than $3 \cdot 10^{-3}$. Furthermore, if we consider that FDT-MAC can operate over an array of directional antennas operating in omnidirectional mode, the AOA precision can reach $1^{\circ}[14,15]$. In this case $(\epsilon=1, \tau=0.1), P_{f}$ value is less than $5 \cdot 10^{-4}$. Thus, the above results show that the source/destination identification accuracy is very high with a negligible probability of error which turns FDT-MAC to be feasible. Next, the results obtained at this work will be presented.

\section{Results}

This section presents the evaluations held in this work. These evaluations aim to highlight the positive impact of the adoption of the proposed scheme. To accomplish this task, the evaluations presented in this section rely on metrics widely used on the related literature. First, an upper bound estimation of throughput considering a scenario with no collisions will be presented, thus allowing the estimation of the maximum throughput. This calculation is commonly employed by related works to compare the performance of medium access control (MAC) techniques [23-25]. Also, based on this metric, a number of works compared different versions of the IEEE 802.11 standard in terms of throughput $[8,26]$.

Although the maximum throughput provides a throughput upper bound for the evaluated MAC technique, it considers a scenario with no collisions leading to an evaluation that does not address the impact of the growth of the backoff time for the network performance. In order to achieve a more accurate estimation of throughput under heavy traffic conditions, many analytical models were proposed in the literature as the presented in [27-30]. These models address the backoff time growth due to collisions, since these models consider that the network is saturated; that is, every node always has packets to transmit, thus contending for the channel. In our work, when this saturation is considered, the throughput will be referred to as channel throughput. It is important to highlight that Bianchi's model [27] is a building block of many other network analytical models [28-30] and widely used to assess the main protocols in the related literature. Many other authors resort to this model to do their analytical performance evaluations [31-33] in half-duplex communications. Simple modifications to the model can be done to extend it to the full-duplex communication scenario, as further detailed in Section 5.2. In this way, Bianchi's model can be used to compare network performance under saturated traffic addressing MAC techniques tailored for halfduplex or full-duplex communications.

Our work will present an analytical evaluation of FDTMAC in the two contexts aforementioned, addressing both collision-free and saturated scenarios. For these evaluations, the widely used throughput calculation for each of these two scenarios (collision-free and saturated) that are proposed in [8] and [27], respectively, will be considered. In order to emphasize the consistence of our calculation, the results obtained in our work are compared with the original values presented in $[8,18,27]$. Furthermore, the impact of selfinterference factor $(K)$ will also be assessed in the performance of full-duplex protocols, thus showing that FDTMAC is able to deal properly with self-interference which is paramount for full-duplex protocols. Moreover, the impact on FDT-MAC of tone suppression and uniform data mode on channel throughput will be discussed. Therefore, our work 
TABLE 2: Constants for IEEE 802.11a and IEEE 802.11b standards.

\begin{tabular}{lcc}
\hline Constant & $802.11 \mathrm{a}$ & $802.11 \mathrm{~b}$ \\
\hline SIFS $\left(T_{\mathrm{SF}}\right)$ & $16 \mu \mathrm{s}$ & $10 \mu \mathrm{s}$ \\
$\mathrm{DIFS}\left(T_{\mathrm{DF}}\right)$ & $34 \mu \mathrm{s}$ & $50 \mu \mathrm{s}$ \\
$C W_{\min }$ & $16 \mu \mathrm{s}$ & $32 \mu \mathrm{s}$ \\
$T_{\text {slot }}$ & $9 \mu \mathrm{s}$ & $20 \mu \mathrm{s}$ \\
$T_{B}=\frac{\left(C W_{\min }-1\right) \cdot T_{\text {slot }}}{2}$ & $67.5 \mu \mathrm{s}$ & $310 \mu \mathrm{s}$ \\
$K$ & 1 & 1 \\
\hline
\end{tabular}

provides a consistent analytical assessment of FDT-MAC by comparing it through widely used models against other traditional MAC techniques. For the referred analysis, it is important to state that the propagation delay of the transmissions was considered negligible in all scenarios. Also, tone suppression and uniform data mode are considered to be enabled only when mentioned.

5.1. Maximum Throughput. This subsection presents the calculus of maximum throughput $\left(S_{\max }\right)$. Maximum throughput calculation is proposed in [8] showing a throughput upper bound of IEEE 802.11b and IEEE 802.11a for varying channel rate and packet size. This calculation was done based on the following definition [8]:

$$
S_{\max }=\frac{8 \cdot P_{\mathrm{sz}}}{T_{t}},
$$

where $P_{\mathrm{sz}}$ denotes the packet payload size and $T_{t}$ denotes the total transmission time. Furthermore, $T_{t}$ has different definitions for HD-MAC, FD-MAC and FDT-MAC as follows:

$$
T_{t}= \begin{cases}L, & \text { for HD-MAC, } \\ L+T_{\mathrm{CT}}+T_{\mathrm{SF}}, & \text { for FD-MAC, } \\ L_{\text {tone }}, & \text { for FDT-MAC }\end{cases}
$$

where

$$
\begin{aligned}
L= & T_{\mathrm{RT}}+T_{\mathrm{CT}}+T_{\mathrm{DATA}}+T_{\mathrm{ACK}}+T_{B}+T_{\mathrm{DF}}+3 \\
& \cdot T_{\mathrm{SF}}, \\
L_{\text {tone }}= & \frac{9}{2} \cdot T_{p}+T_{\mathrm{DATA}}+T_{B}+T_{\mathrm{DF}}+\frac{9}{2} \cdot T_{\mathrm{SF}} .
\end{aligned}
$$

Based on (3), $S_{\max }$ was calculated for HD-MAC, FD$\mathrm{MAC}$, and FDT-MAC. In these calculations, it was considered that communications of type 1 and type 2 (defined in Section 3) are equiprobable. Also, it was considered that all the communications have the same payload size. Moreover, the calculation was done for parameters of the IEEE 802.11a and IEEE $802.11 \mathrm{~b}$ standards [7]. The values of some constants related to these standards are available in Table 2.

The Table 3 shows the results of the $S_{\max }$ comparison of FDT-MAC against HD-MAC and FD-MAC for various values of payload size and channel rate. By observing Table 3 , it can be seen that FDT-MAC can provide a significant improvement of throughput up to $220.62 \%$ against HD-MAC

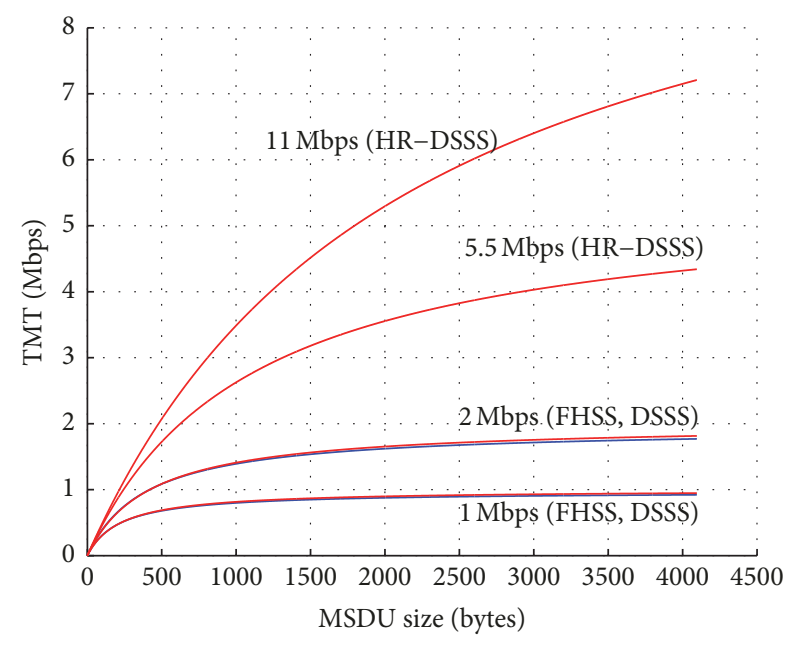

Figure 6: Values of $S_{\max }$ for IEEE $802.11 \mathrm{~b}$ calculated in [8].

and up to $84.54 \%$ against FD-MAC. Note also that FDTMAC outperforms FD-MAC in all scenarios. As the payload transmitted by FD-MAC and FDT-MAC is the same, this difference occurs due to the time spent to accomplish a communication $\left(T_{t}\right)$. For FDT-MAC, $T_{t}$ is always lower than for FD-MAC, since $L_{\text {tone }} \leq L+T_{\mathrm{CT}}+T_{\mathrm{SF}}$ occurs in all evaluated scenarios of Table 3 . The gain of FDT-MAC is higher when the latency of channel reservation constitutes a higher portion of the total transmission time, once $T_{\text {DATA }}$ is the same for both FD-MAC and FDT-MAC. Also, it is remarkable that FDT-MAC outperforms HD-MAC in more than $100 \%$ in most of the cases. This means that FDT-MAC can exploit the theoretical advantage of using a full-duplex antenna, since full-duplex antenna is expected to provide twice as much throughput.

In order to emphasize the consistency of the presented calculation, the HD-MAC values in Table 3 will be compared with the original values calculated in [8] for both IEEE 802.11a and IEEE $802.11 \mathrm{~b}$. The original values calculated by [8] are displayed in Figures 6 and 7. Figure 6 presents the values of maximum throughput calculated by [8] for IEEE $802.11 \mathrm{~b}$ operating in half-duplex antennas, while Figure 7 presents the values for IEEE 802.11a. Note that the values calculated for HD-MAC in our work (Table 3 ) are very close to the presented in Figures 6 and 7. For instance, for the channel rate of $2 \mathrm{Mbps}$ and the payload size of 512 bytes, it can be observed in Figure 6 that maximum throughput value is slightly higher than 1 Mbps. Furthermore, in Table 3 the value calculated is $1.16 \mathrm{Mbps}$ which is very close to the expected. Another example is the case of channel rate equal to $6 \mathrm{Mbps}$ and payload size of 1024 bytes. As presented in Figure 7, the value expected shall be slightly lower than $5 \mathrm{Mbps}$. Note that this is consistent with Table 3 that calculated maximum throughput to be equal to $4.75 \mathrm{Mbps}$ in this scenario. A small difference on the values is expected to occur for IEEE 802.11a, once the calculation presented by [8] considered SIFS time to be equal to $9 \mu \mathrm{s}$, while our work considers this value to be equal to $16 \mu \mathrm{s}$ as stated in the IEEE 802.11a standard [7]. 
TABLE 3: Maximum throughput for various protocols.

\begin{tabular}{|c|c|c|c|c|c|c|c|}
\hline Scheme & $R_{c}(\mathrm{Mbps})$ & $P_{\mathrm{sz}}$ (bytes) & $\begin{array}{c}\text { FDT-MAC } \\
S_{\max }(\mathrm{Mbps})\end{array}$ & $\begin{array}{c}\text { HD-MAC } \\
S_{\max }(\mathrm{Mbps})\end{array}$ & Gain (\%) & $\begin{array}{c}\text { FD-MAC } \\
S_{\max }(\mathrm{Mbps})\end{array}$ & Gain (\%) \\
\hline \multirow{6}{*}{ DSSS } & \multirow{3}{*}{1} & 256 & 1.38 & 0.53 & 159.59 & 0.98 & 40.35 \\
\hline & & 512 & 1.63 & 0.69 & 135.29 & 1.32 & 23.90 \\
\hline & & 1024 & 1.80 & 0.82 & 119.44 & 1.59 & 13.16 \\
\hline & \multirow{3}{*}{2} & 256 & 2.25 & 0.82 & 175.82 & 1.48 & 52.09 \\
\hline & & 512 & 2.88 & 1.16 & 148.52 & 2.16 & 33.33 \\
\hline & & 1024 & 3.35 & 1.47 & 128.21 & 2.81 & 19.38 \\
\hline \multirow{3}{*}{ HR } & \multirow{3}{*}{11} & 256 & 4.68 & 1.46 & 220.62 & 2.54 & 84.54 \\
\hline & & 512 & 7.72 & 2.58 & 199.44 & 4.55 & 69.71 \\
\hline & & 1024 & 11.43 & 4.18 & 173.61 & 7.54 & 51.60 \\
\hline \multirow{9}{*}{ OFDM } & \multirow{3}{*}{6} & 256 & 6.36 & 2.92 & 117.86 & 5.38 & 18.24 \\
\hline & & 512 & 8.33 & 3.93 & 111.69 & 7.44 & 11.94 \\
\hline & & 1024 & 9.82 & 4.75 & 106.89 & 9.18 & 7.04 \\
\hline & \multirow{3}{*}{12} & 256 & 9.05 & 4.40 & 105.74 & 7.98 & 13.48 \\
\hline & & 512 & 13.12 & 6.43 & 104.16 & 11.95 & 9.77 \\
\hline & & 1024 & 16.99 & 8.38 & 102.70 & 15.98 & 6.32 \\
\hline & \multirow{3}{*}{54} & 256 & 13.43 & 7.17 & 87.21 & 12.58 & 6.72 \\
\hline & & 512 & 23.74 & 12.58 & 88.70 & 22.41 & 5.94 \\
\hline & & 1024 & 38.92 & 20.40 & 90.74 & 37.11 & 4.87 \\
\hline
\end{tabular}

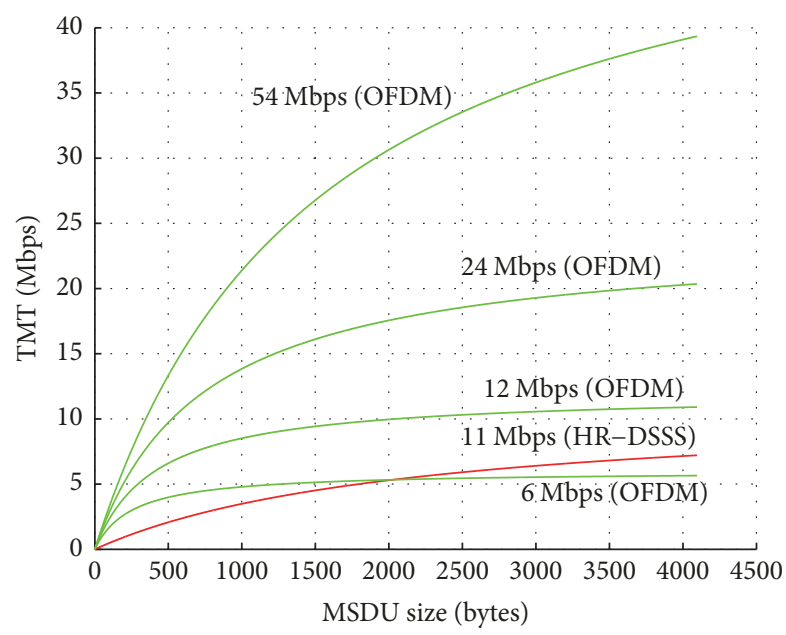

FIgURE 7: Values of $S_{\max }$ for IEEE 802.11a calculated in [8].

Therefore, a consistent calculation of an upper bound in terms of throughput for FDT-MAC, FD-MAC, and HDMAC was presented. The maximum throughput of FDTMAC outperformed FD-MAC in all scenarios being up to $84.54 \%$ higher. Also, in most of cases maximum throughput of FDT-MAC surpassed HD-MAC in more than 100\%, thus indicating that FDT-MAC may properly exploit the potential of full-duplex communications. As previously explained, the maximum throughput does not address the losses due to collisions. Next, an analytical evaluation which deals with the referred situation will be presented.

5.2. Channel Throughput. In order to assess the impact of backoff growth due to collisions in the throughput of FDTMAC, FD-MAC, and HD-MAC, our work uses the wellknown Bianchi's model [27]. This model considers that the network is saturated; that is, every node always has packets to transmit, thus contending for the channel. Due to these conditions, in this evaluation, the throughput is referred to as channel throughput. Bianchi's model is used in many articles to evaluate network performance and compare MAC schemes as presented in [31-33]. Furthermore, other models were designed to estimate channel throughput. These models mainly rely on Bianchi's model with few changes as described in [28-30]. Due to its relevance and common use in the related literature, Bianchi's model was chosen to the evaluations of channel throughput that are presented in our work. This model was tailored and mainly used to assess techniques based on half-duplex antennas. However, this model is also suitable to techniques based on full-duplex antennas, since it relies on the fact that a transmission is successful if only one of the nodes' backoff counter has reached zero at a given time. Once this constraint is still valid for full-duplex antennas, it makes this model feasible to evaluate techniques of both antenna operation modes, as done in the evaluations of the original paper of FD-MAC presented in $[5,18]$.

Bianchi's model mathematical definition of channel throughput $(S)$ is [27]

$$
S=\frac{\left(p_{s} \cdot p_{\mathrm{tr}} \cdot P_{\mathrm{sz}}\right)}{\bar{T}_{\mathrm{slot}}}
$$

where

$$
\begin{aligned}
\bar{T}_{\text {slot }}= & T_{\text {slot }} \cdot\left(1-p_{\text {tr }}\right)+p_{\text {tr }} \cdot p_{s} \cdot T_{s}+p_{\text {tr }} \cdot\left(1-p_{s}\right) \\
& \cdot T_{c}, \\
T s= & T_{t}-T_{B}, \\
T c= & T_{\mathrm{RT}}+T_{\mathrm{DF}},
\end{aligned}
$$

$p_{\text {tr }}$ denotes the probability of at least one transmission occurring during a time slot and $p_{s}$ denotes the probability of a transmission occurring during a time slot be successful. 
TABLE 4: Parameters used for throughput evaluation.

\begin{tabular}{lc}
\hline$R_{c}$ (for all transmissions) & $1 \mathrm{Mbps}$ \\
\hline$P_{\mathrm{sz}}^{i j}(\forall i, j)$ & $8184 \mathrm{bits}$ \\
$T_{\mathrm{DF}}$ & $128 \mu \mathrm{s}$ \\
$T_{\mathrm{SF}}$ & $28 \mu \mathrm{s}$ \\
$K$ & 1 \\
\hline
\end{tabular}

These probabilities are dependent of the parameters of exponential backoff algorithm (minimum and maximum backoff window size) and of the amount of nodes in the network. For further details on the calculation of these probabilities, the interested reader is directed to [27] and the references within.

There is only a change needed to (6) to couple full-duplex antennas communication. When a successful communication occurs, the total payload $\left(P_{\mathrm{sz}}\right)$ must be the sum of the payloads of both data communication. This change is due to the fact that data can be sent from $A$ to $B$ and from $B$ to $A$ in the same successful communication, as depicted in Figure 1(b). So, for all results presented in this paper the channel throughput is considered as follows:

$$
\begin{aligned}
S & =\frac{\left(p_{s} \cdot p_{\mathrm{tr}} \cdot P_{\text {szexp }}\right)}{\bar{T}_{\text {slot }}}, \\
P_{\text {szexp }} & =P_{\text {sz }}^{a b}+P_{s z}^{b a},
\end{aligned}
$$

where $P_{\mathrm{sz}}^{i j}$ denotes the payload of the data transmitted from node $i$ to $j$. Recall that FDT-MAC employs pulse/tone signals instead of RTS/CTS frames. Therefore, $T_{c}=T_{\text {pulse }}+T_{\mathrm{DF}}$ for FDT-MAC. Note that $T_{c}$ is lower for FDT-MAC than for FDMAC, once $T_{\text {pulse }}+T_{\mathrm{DF}}<T_{\mathrm{RT}}+T_{\mathrm{DF}}$ because $T_{\text {pulse }}<T_{\mathrm{RT}}$. Also, it can be observed that $T_{s}$ is lower for FDT-MAC than for FD-MAC, once $T_{t}$ is lower for FDT-MAC as previously discussed in Section 5.1. So, as both $T_{c}$ and $T_{s}$ are lower for FDT-MAC than for FD-MAC, it is expected that $\bar{T}_{\text {slot }}$ is lower for FDT-MAC. According to (8), this causes FDT-MAC to be expected to have a higher channel throughput $(S)$ than FDMAC. Indeed, this expectation is confirmed to be valid as presented next.

From (8) and (9), the throughput for various number of nodes was calculated when using HD-MAC, FD-MAC, and FDT-MAC schemes. For this calculation, the parameters used in Table 4 were used, since they are the same used in [5, $18,27]$. The size of physical and media access control layer headers as well as backoff window parameters were the same as the defined in IEEE 802.11b standard. The comparison of FDT-MAC against HD-MAC and FD-MAC is presented in Figure 8. As can be observed, FDT-MAC outperformed FD-MAC and HD-MAC in up to $156 \%$ and up to $412 \%$, respectively. Note that the advantage of the use of FDT-MAC increases as the number of nodes rises, once FDT-MAC has a lower latency being able to couple with saturated environments much better than FD-MAC and HD-MAC do. Also, FDT-MAC takes advantage of the full-duplex mode of the antenna, once FDT-MAC outperforms HD-MAC in more than $100 \%$ for all the evaluated cases. Observe that in this

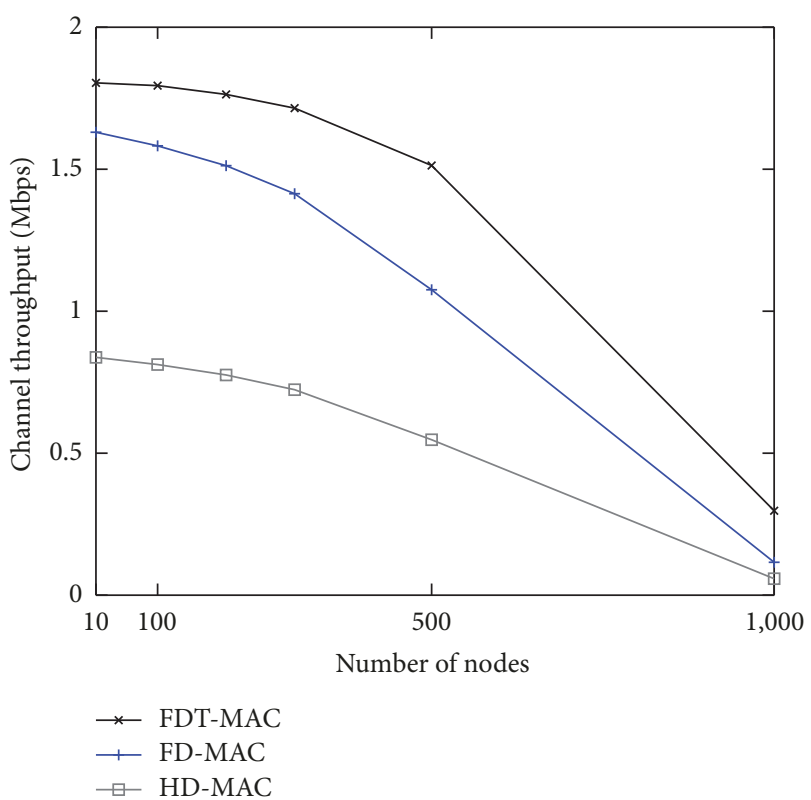

FIGURE 8: Scheme comparison regarding channel throughput.

evaluation it was considered that $P_{\mathrm{sz}}=8184$ bits and $R_{c}=$ $1 \mathrm{Mbps}$; that is, $T_{\text {DATA }}$ represents a great portion of total transmission time. If a lower packet size and/or a higher transmission channel rate is considered, the gain of FDT-MAC would be still higher, since in these cases channel reservation latency would have more impact over network throughput. Furthermore, it can be observed that for $n=10$ the channel throughput is close to the maximum throughput calculated in the Section 5.1. However, channel throughput never attains values higher than maximum throughput once maximum throughput is calculated in a collision-free scenario. Moreover, note that as the number of nodes increases the channel throughput dramatically reduces for the three evaluated MAC schemes (FDT-MAC, FD-MAC, and HD-MAC). This happens since the higher the number of nodes, the higher the probability of collisions. When the amount of collisions grows, the time spent with backoff rises, thus reducing channel throughput.

Also, the channel throughput values calculated for HD$\mathrm{MAC}$ and FD-MAC will be compared with the original values calculated for these MAC schemes that are available in [27] and [18], respectively. This comparison is done to show the consistency of the calculations held in our work, once the calculated values of HD-MAC and FD-MAC are very close to the original ones presented in $[18,27]$. By these means, it is paramount to emphasize that FDT-MAC really outperforms the other schemes in terms of channel throughput, since our results are solid and consistent with the related literature as shown next. Figure 9 shows the original values calculated for FD-MAC and HD-MAC available in [18]. Note that these values are very similar to the values calculated in our work (Figure 8). However, in their results, they use throughput versus probability $p$ instead of the number of nodes. The $n$ depicted in Figure 9 denotes the number of nodes and their $p$ has the same meaning that $\sigma=1-\sqrt[n]{p_{\mathrm{tr}}-1}$, where $p_{\mathrm{tr}}$ is 


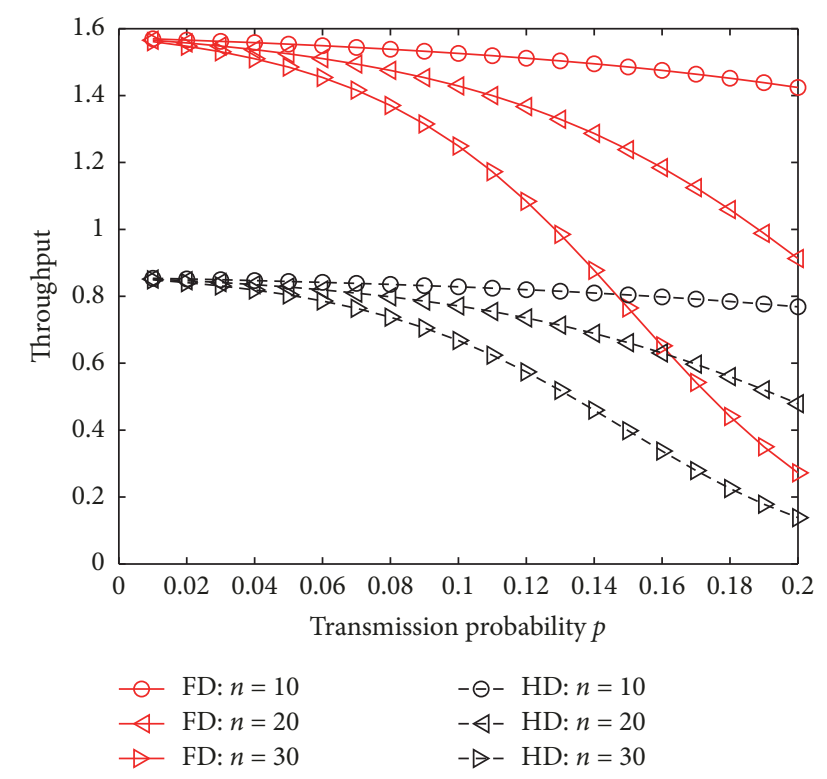

Figure 9: Original values for FD-MAC of channel throughput versus transmission probability calculated in [18].

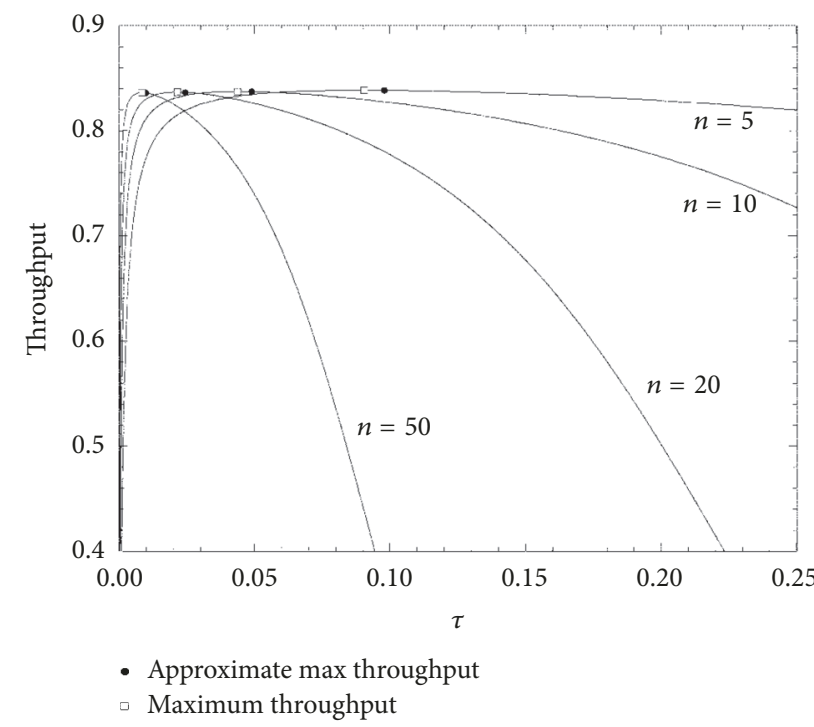

FIGURE 10: Original values for HD-MAC of channel throughput versus transmission $\tau$ calculated in [27].

the same used in (6). An example of comparison will be presented next. In our calculations, for $n=10, p_{\text {tr }}$ is equal to 0.32 and $\sigma=0.038$. Note that the channel throughput values for both FD-MAC and HD-MAC for $p=0.038$ in Figure 9 are very close to the presented values in Figure 8 for $n=10$. In both figures, observe that HD-MAC channel throughput value is slightly higher than $0.8 \mathrm{Mbps}$ while FDMAC channel throughput value almost reaches $1.6 \mathrm{Mbps}$. Therefore, it was shown that our calculation is consistent with the one presented in [18]. Now, the channel throughput value calculated for HD-MAC will be compared with the original values presented by Bianchi in [27]. The original channel throughput values calculated in Bianchi model for HD-MAC are illustrated in Figure 10. However, channel throughput is plotted versus probability $\tau$ instead of the number of nodes. It is important to state that in [27] $\tau$ has the same meaning that $\sigma=1-\sqrt[n]{p_{\mathrm{tr}}-1}$. To perform a comparison, recall that, for $n=10, \sigma=0.038$. Moreover, it can be observed that the throughput value for $\tau=0.038$ (curve $n=10$ ) in Figure 10 is slightly higher than 0.8 which is very close to the one presented for $n=10$ in Figure 8, where channel throughput is equal to $0.83 \mathrm{Mbps}$. Again, the calculation of channel throughput is shown to be solid with the original values presented in the related works $[18,27]$. This concludes the analysis of the impact of FDT-MAC over channel throughput and about the consistency of our results. Next, the impact of self-interference on full-duplex communications will be evaluated based on the concept of channel throughput proposed in [27] and explained in the current subsection.

5.3. Impact of Self-Interference Factor. As explained in Section 2 the self-interference may diminish the network performance. In order to evaluate this impact, analysis was held over FD-MAC and FDT-MAC to show how they perform under different values of self-interference factor $(K)$. This analysis was not done for HD-MAC, since it does not suffer from selfinterference as explained in Section 2. The presented analysis will only address the case where the network is considered to be saturated and impacted by backoff growth caused by collisions. We will not address the collision-free case because the calculation of the maximum throughput is trivial for selfinterference. In this case, the maximum throughput expected would be equal to $K \cdot S_{\max }$ for both FDT-MAC and FDMAC. So, the percentage of the difference of the maximum throughput values would tend to be very similar to the presented in Section 5.1 (Table 3).

Therefore, to conduct the evaluation in terms of channel throughput it was needed to change (9) to address the impact of $K$ in the Bianchi model. Recall the definition of types 1 and 2 of communications presented in Section 3. The modification to (9) was related to the estimated frame size, in order to differ between types 1 and 2 of full-duplex communications depicted in Figure 1. This differentiation was made because type 1 is more affected than type 2 by selfinterference, whereas type 1 has two antennas transmitting and receiving simultaneously, while type 2 has only one. So, for this evaluation, (9) is replaced by the following one:

$$
\begin{aligned}
& P_{\text {szexp }}=2 \cdot\left(\beta \cdot K \cdot\left(P_{\mathrm{sz}}^{a b}+P_{\mathrm{sz}}^{b a}\right)+(1-\beta) \cdot K^{2}\right. \\
& \left.\cdot\left(P_{\mathrm{sz}}^{a b}+P_{\mathrm{sz}}^{b a}\right)\right),
\end{aligned}
$$

where $\beta(0 \leq \beta \leq 1)$ denotes the proportion of occurrences of bidirectional links (showed in Figure 1(b)) in the network. Again, it is considered that types 1 and 2 of the communications are equiprobable $(\beta=0.5)$. The other parameters used to this evaluation are the presented in Table 4. Therefore, it was possible to obtain results about the impact of $K$ for both FDT-MAC and FD-MAC. These results are presented in Table 5. FDT-MAC outperforms FDMAC again in all cases in up to $236 \%$. It is important to note that the higher the number of nodes, the higher the gain 
TABLE 5: Impact of self-interference factor on throughput.

\begin{tabular}{|c|c|c|c|c|}
\hline K & Nodes & $\begin{array}{c}\text { FDT-MAC } \\
S_{\max }(\mathrm{Mbps})\end{array}$ & $\begin{array}{c}\text { FD-MAC } \\
S_{\max }(\mathrm{Mbps})\end{array}$ & Gain (\%) \\
\hline \multirow{6}{*}{1} & 10 & 1.80 & 1.63 & 10.66 \\
\hline & 100 & 1.79 & 1.58 & 13.40 \\
\hline & 200 & 1.76 & 1.51 & 16.59 \\
\hline & 300 & 1.72 & 1.41 & 21.31 \\
\hline & 500 & 1.51 & 1.08 & 40.69 \\
\hline & 1000 & 0.30 & 0.12 & 156.6 \\
\hline \multirow{6}{*}{0.95} & 10 & 1.67 & 1.51 & 11.03 \\
\hline & 100 & 1.66 & 1.46 & 13.93 \\
\hline & 200 & 1.63 & 1.39 & 17.39 \\
\hline & 300 & 1.59 & 1.30 & 22.50 \\
\hline & 500 & 1.40 & 0.98 & 43.51 \\
\hline & 1000 & 0.28 & 0.10 & 169.2 \\
\hline \multirow{6}{*}{0.9} & 10 & 1.54 & 1.38 & 11.43 \\
\hline & 100 & 1.53 & 1.34 & 14.53 \\
\hline & 200 & 1.51 & 1.27 & 18.27 \\
\hline & 300 & 1.47 & 1.18 & 23.83 \\
\hline & 500 & 1.29 & 0.88 & 46.65 \\
\hline & 1000 & 0.25 & 0.09 & 183.21 \\
\hline \multirow{6}{*}{0.85} & 10 & 1.42 & 1.27 & 11.88 \\
\hline & 100 & 1.41 & 1.22 & 15.19 \\
\hline & 200 & 1.39 & 1.16 & 19.26 \\
\hline & 300 & 1.35 & 1.08 & 25.31 \\
\hline & 500 & 1.19 & 0.79 & 50.15 \\
\hline & 1000 & 0.23 & 0.08 & 198.86 \\
\hline \multirow{6}{*}{0.8} & 10 & 1.30 & 1.16 & 12.38 \\
\hline & 100 & 1.29 & 1.11 & 15.94 \\
\hline & 200 & 1.27 & 1.05 & 20.38 \\
\hline & 300 & 1.23 & 0.97 & 26.98 \\
\hline & 500 & 1.09 & 0.71 & 54.1 \\
\hline & 1000 & 0.21 & 0.07 & 216.47 \\
\hline \multirow{6}{*}{0.75} & 10 & 1.18 & 1.05 & 12.96 \\
\hline & 100 & 1.18 & 1.01 & 16.79 \\
\hline & 200 & 1.16 & 0.95 & 21.64 \\
\hline & 300 & 1.13 & 0.87 & 28.86 \\
\hline & 500 & 0.99 & 0.63 & 58.57 \\
\hline & 1000 & 0.20 & 0.06 & 236.43 \\
\hline
\end{tabular}

of FDT-MAC over FD-MAC. Also, the referred gain rises as $K$ decreases. These behaviors occur because under these situations the latency to make channel reservation is more critic. This higher criticality takes place because more communications are tried to be established, either by the rise of the amount of nodes or by the increasing of losses due to selfinterference factor $(K)$ decrease.

Although $K$ has impact over the network performance, there are several techniques that almost cancel it [10]. So, many of the related works of full-duplex antennas consider $K$ value to be very close to 1 [5]. In this case $(K=1)$, the results of FD-MAC and FDT-MAC for channel throughput are the same already presented in Section 5.2. Therefore, FDTMAC attains higher throughput than FD-MAC either in the scenario with no self-interference or in the critic scenario of a rising self-interference.

5.4. Tone Suppression. As explained in Section 4, FDT-MAC realizes tone suppression in situations where an extra tone is unnecessary due to the packet payload size. Recall that $P_{\mathrm{sz}}^{i j}$ denotes the payload of the data transmitted from node $i$ to $j$. In order to assess the impact of tone suppression, the following scenarios have been evaluated:

(I) $P_{\mathrm{sz}}^{A B}=P_{\mathrm{sz}}^{B A}=256$ bytes; 
TABLE 6: Maximum throughput with tone suppression.

\begin{tabular}{|c|c|c|c|c|c|}
\hline Scheme & $R_{c}(\mathrm{Mbps})$ & Scenario & $\begin{array}{c}\text { FDT-MAC } \\
S_{\max }(\mathrm{Mbps})\end{array}$ & $\begin{array}{c}\text { FD-MAC } \\
S_{\max }(\mathrm{Mbps})\end{array}$ & Gain (\%) \\
\hline \multirow{6}{*}{ DSSS } & \multirow{3}{*}{1} & I & 1.39 & 0.98 & 41.99 \\
\hline & & II & 1.23 & 0.99 & 24.75 \\
\hline & & III & 1.35 & 1.19 & 13.59 \\
\hline & \multirow{3}{*}{2} & I & 2.30 & 1.48 & 55.24 \\
\hline & & II & 2.19 & 1.62 & 35.09 \\
\hline & & III & 2.53 & 2.10 & 20.29 \\
\hline \multirow{3}{*}{ HR } & \multirow{3}{*}{11} & I & 4.90 & 2.54 & 93.04 \\
\hline & & II & 6.01 & 3.41 & 76.10 \\
\hline & & III & 8.81 & 5.66 & 55.78 \\
\hline \multirow{9}{*}{ OFDM } & \multirow{3}{*}{6} & I & 6.82 & 5.38 & 26.81 \\
\hline & & II & 6.53 & 5.58 & 17.12 \\
\hline & & III & 7.56 & 6.88 & 9.91 \\
\hline & \multirow{3}{*}{12} & I & 10.08 & 7.98 & 26.32 \\
\hline & & II & 10.62 & 8.96 & 18.50 \\
\hline & & III & 13.38 & 11.98 & 11.65 \\
\hline & \multirow{3}{*}{54} & I & 15.91 & 12.58 & 26.41 \\
\hline & & II & 20.65 & 16.81 & 22.86 \\
\hline & & III & 32.90 & 27.83 & 18.21 \\
\hline
\end{tabular}

(II) $P_{\mathrm{sz}}^{A B}=512$ bytes, $P_{\mathrm{sz}}^{B A}=256$ bytes;

(III) $P_{\mathrm{sz}}^{A B}=1024$ bytes, $P_{\mathrm{sz}}^{B A}=512$ bytes.

These scenarios were evaluated through maximum throughput $\left(S_{\max }\right.$ defined in Section 5.1) and channel throughput ( $S$ defined in Section 5.2) metrics; that is, this analysis addresses the collision-free and the heavy traffic network situations. Also, it was considered that all the links are bidirectional $(\beta=1)$. Due to this behavior and assuming that for all communications in the scenarios listed above the tone suppression occurs, $L_{\text {tone }}$ equation was changed for FDTMAC. Once the tone-c sent from $A$ is suppressed (Figure 3), $L_{\text {tone }}$ is defined as follows:

$$
L_{\text {tone }}=3 \cdot T_{p}+T_{\mathrm{DATA}}+T_{B}+T_{\mathrm{DF}}+3 \cdot T_{\mathrm{SF}} \cdot
$$

So, $S_{\max }$ was calculated according to (3) and (11). The results of this calculation are displayed at Table 6 . The results point a higher throughput for FDT-MAC in all cases. The throughput gain is at least of $9 \%$ and up to $93 \%$ in the evaluated scenarios. Note that the results of FDT-MAC for scenario $1\left(P_{\mathrm{sz}}^{A B}=P_{\mathrm{sz}}^{B A}=256\right.$ bytes $)$ can be compared to $S_{\max }$ calculated without considering tone suppression (Table 3 ). This evaluation is presented in Table 7 analyzing FDT-MAC with tone suppression against FDT-MAC without it. By observing Table 7 , it can be seen that tone suppression can achieve a gain of throughput up to $18 \%$. Also, it is remarkable that for OFDM scheme (used by IEEE 802.11a) the impact of tone suppression is much higher. Therefore, it can be concluded that tone suppression plays an important role on FDT-MAC, once it allows a faster channel reservation for some situations as described in Section 4.

Furthermore, tone suppression was also evaluated through channel throughput defined by Bianchi [27]. With the aid of (8) and (11), the channel throughput $(S)$ for various number of nodes $(10,20,30,40,50)$ considering
FD-MAC and FDT-MAC schemes was calculated. This comparison was held for considering both IEEE 802.11a and IEEE 802.11 b physical layer parameters. The size of physical and media access control layer headers and backoff window parameters were the same as the defined in the standard original versions. Some of these parameters are described in Table 2. For more details on these parameters and these versions of the standard, the reader is directed to [7]. For the analysis that consider IEEE $802.11 \mathrm{~b}$ physical layer scheme, the channel rate was considered to be equal to $11 \mathrm{Mbps}$, while it was considered to be equal to $54 \mathrm{Mbps}$ for IEEE 802.11a physical layer scheme. The packet size is varied according to the three scenarios (I, II, and III) previously listed, which are the same presented in Table 7. The results for IEEE 802.11a and IEEE $802.11 \mathrm{~b}$ physical layer schemes are presented in Figures 11 and 12, respectively. The three scenarios of packet size are shown in each one of the figures. The identifier of the scenario is indicated in the legend of the figure after the name of the MAC technique. Note that, in all the three scenarios, FDT-MAC dramatically surpassed FD-MAC channel throughput. The gain was at least of $20 \%$ and rises up to $35 \%$ when considering the IEEE 802.1la physical layer parameters. In turn, when considering the IEEE 802.11b physical layer parameters, the gain of FDT-MAC was at least of $41 \%$, reaching $79 \%$ at most. A fast channel reservation is highly desirable, especially in intensive traffic environments as the considered in our analysis. Indeed, the presented analysis points that FDT-MAC combined with tone suppression is able to achieve channel reservation much faster than FD-MAC, thus leading to relevant improvement in terms of throughput, as indicated in Table 6 and Figures 11 and 12 .

5.5. Uniform Data. Recall from Section 4 that FDT-MAC can enable the uniform data mode. This mode changes the 
TABLE 7: Impact of tone suppression.

\begin{tabular}{lcccc}
\hline Scheme & $R_{c}$ (Mbps) & \multicolumn{2}{c}{ Tone suppression } & \\
& & $S_{\max }(\mathrm{Mbps})$ & $S_{\max }(\mathrm{Mbps})$ & \\
\hline \multirow{2}{*}{ DSSS } & 1 & 1.39 & 1.38 & 0.72 \\
& 2 & 2.30 & 2.25 & 2.22 \\
\hline HR & 11 & 4.90 & 4.68 & 4.70 \\
\hline \multirow{2}{*}{ OFDM } & 6 & 6.82 & 6.36 & 7.23 \\
& 12 & 10.08 & 9.05 & 11.38 \\
& 54 & 15.91 & 13.43 & $\mathbf{1 8 . 4 7}$ \\
\hline
\end{tabular}

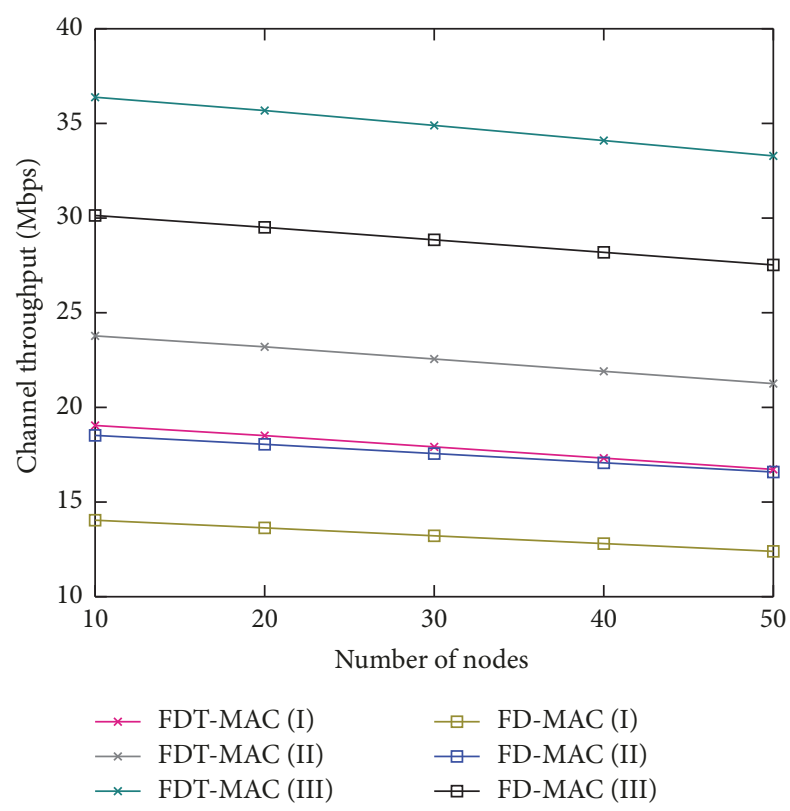

FIGURE 11: Channel throughput for tone suppression and IEEE 802.11a with $R_{c}=54 \mathrm{Mbps}$.

behavior of FDT-MAC by defining DATA as $\min (\mathrm{DATA}(A$, $B), \operatorname{DATA}(B, A))$, instead of $\max (\operatorname{DATA}(A, B), \operatorname{DATA}(B, A))$. This causes the scheduling of a new communication to transmit the remaining data of size equal to $\max (\operatorname{DATA}(A$, $B), \operatorname{DATA}(B, A))-\min (\operatorname{DATA}(A, B), \operatorname{DATA}(B, A))$. Note that, in the second communication, one of the nodes will be only receiving DATA being free to start a new communication with a third node, since every node is considered to be equipped with full-duplex antenna. Therefore, uniform data mode aims to improve both throughput and channel use. The impact of this mode on throughput will be evaluated in two different ways:

(1) Throughput calculation over a simple scenario used to exemplify the relevance of uniform data mode.

(2) Throughput calculation for a scenario with many nodes with heavy traffic.

The first evaluation is based on a simple two-node ( $A$ and $B$ ) scenario described next. Node $A$ has two packets with

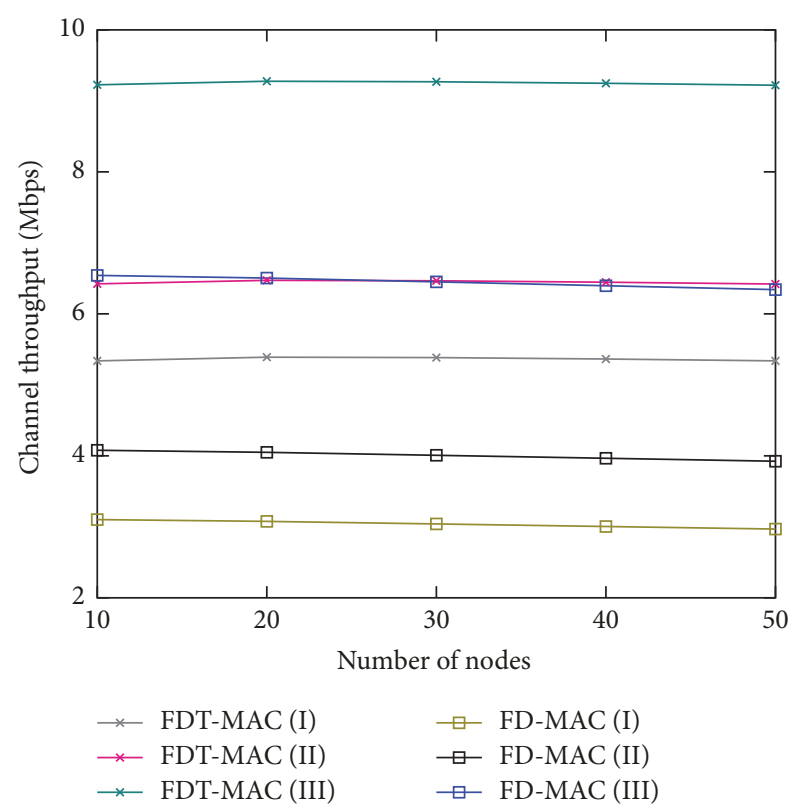

FIGURE 12: Channel throughput for tone suppression and IEEE 802.11b with $R_{c}=11 \mathrm{Mbps}$.

payload of 512 bytes each to transmit to node $B$. Furthermore, node $B$ has a packet with payload of 1024 bytes to transmit to node $A$. Both nodes $A$ and $B$ are equipped with full-duplex antennas. The evaluation compares the following MAC schemes: FD-MAC, FDT-MAC, and FDT-MAC with uniform mode. As all of these schemes need to perform two communications to accomplish successfully the delivery of the described payload, $T_{t}(1)$ denotes the time spent with the first communication, while $T_{t}(2)$ denotes the time spent with the second one. The total transmission time is denoted as $T_{t}$ and is equal to the sum of the transmission time of the two communications $\left(T_{t}=T_{t}(1)+T_{t}(2)\right)$. The PHY schemes considered were IEEE 802.11b and IEEE 802.11a with data rate of $1 \mathrm{Mbps}$ and $6 \mathrm{Mbps}$, respectively. As collisions are not addressed in this scenario, the maximum throughput metric defined in [8] and detailed in Section 5.1 was used. So, the throughput was calculated for the aforementioned parameters with the aid of (3). The results of the comparisons are displayed in Table 8. As can be observed, uniform data mode achieves a throughput $41.38 \%$ and $27.98 \%$ higher than FDT-MAC in IEEE 802.11b and IEEE 802.11a, respectively. When compared with FD-MAC, the gain is of $65.66 \%$ and $51.06 \%$ in IEEE $802.11 \mathrm{~b}$ and IEEE 802.11a, respectively. Therefore, the impact of uniform data mode along with FDT-MAC in this scenario is dramatically positive. However, it is necessary to evaluate uniform data mode also under general condition to allow a more accurate evaluation of its impact.

FDT-MAC uniform data mode was also evaluated using Bianchi's model [27] (detailed in Section 5.2) to allow an assessment under general condition and critical conditions, such as heavy traffic when the amount of nodes increases. In this evaluation, the channel throughput of FDT-MAC will be compared with uniform mode against FDT-MAC without it. 


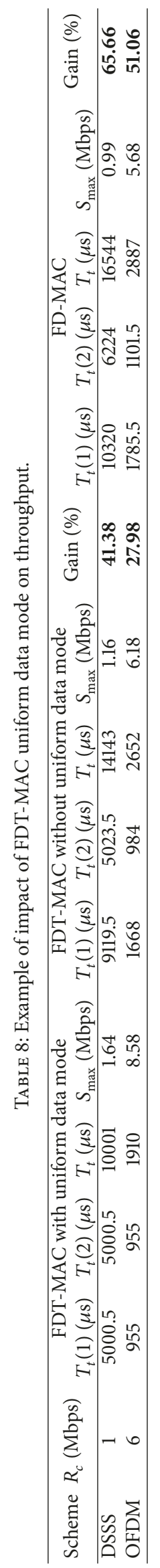




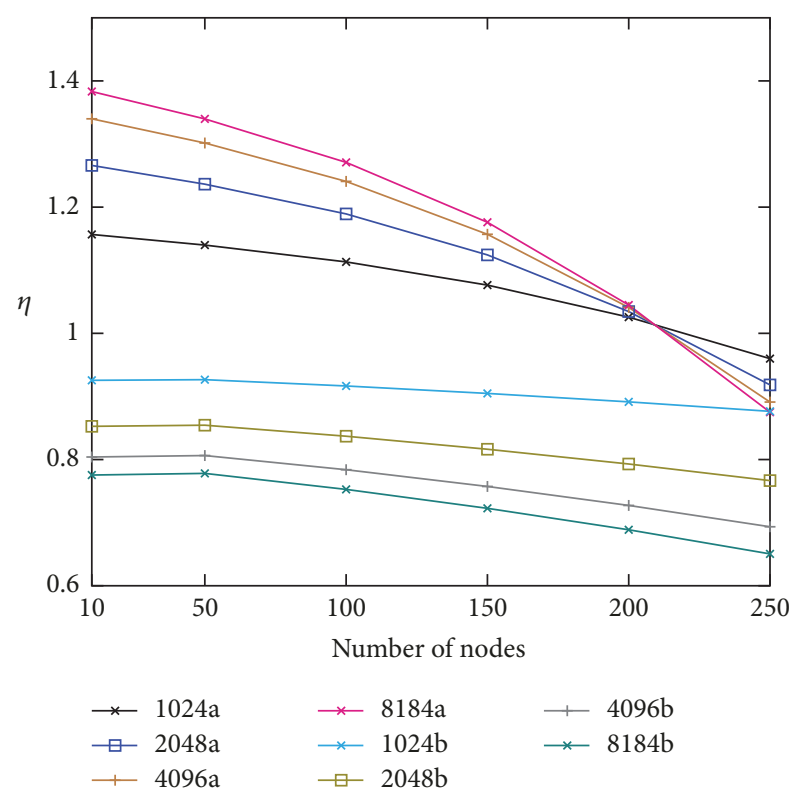

FIGURE 13: Uniform mode throughput comparison when $P_{\mathrm{sz}}(1)=$ 512.

The evaluation was held assuming the use of IEEE $802.11 \mathrm{~b}$ and IEEE 802.11a with data rates of $1 \mathrm{Mbps}$ and $6 \mathrm{Mbps}$, respectively. The number of nodes was varied along the following values: $10,50,100,150,200$, and 250. For this evaluation, FDT-MAC is considered to have always two ongoing data flows. $P_{\mathrm{sz}}(1)$ and $P_{\mathrm{sz}}(2)$ denote the payload of these two flows in bytes. In this analysis, it was assumed that the payload of these flows is different $\left(P_{\mathrm{sz}}(1)<P_{\mathrm{sz}}(2)\right)$. The values of $P_{\mathrm{sz}}(1)$ and $P_{\mathrm{sz}}(2)$ were varied according to the following combinations:

(1) $P_{\mathrm{sz}}(1)=512, P_{\mathrm{sz}}(2)=1024$;

(2) $P_{\mathrm{sz}}(1)=512, P_{\mathrm{sz}}(2)=2048$;

(3) $P_{\mathrm{sz}}(1)=512, P_{\mathrm{sz}}(2)=4096$;

(4) $P_{\mathrm{sz}}(1)=512, P_{\mathrm{sz}}(2)=8184$;

(5) $P_{\mathrm{sz}}(1)=1024, P_{\mathrm{sz}}(2)=2048$;

(6) $P_{\mathrm{sz}}(1)=1024, P_{\mathrm{sz}}(2)=4096$;

(7) $P_{\mathrm{sz}}(1)=1024, P_{\mathrm{sz}}(2)=8184$;

(8) $P_{\mathrm{sz}}(1)=2048, P_{\mathrm{sz}}(2)=4096$;

(9) $P_{\mathrm{sz}}(1)=2048, P_{\mathrm{sz}}(2)=8184$.

The results of the scenarios that $P_{\mathrm{sz}}(1)=512$ are depicted in Figure 13. For various amount of nodes, Figure 13 presents the quotient between the throughput achieved with uniform data mode $\left(S^{\prime}\right)$ and the throughput achieved by FDT-MAC without uniform data mode $(S)$. This quotient $\left(\eta=S^{\prime} / S\right)$ reveals the percentage gain $(\eta>1)$ or loss $(\eta<1)$ from the uniform data mode employment in the network in saturated conditions. Lines that end with "a" at the legend entry were calculated with IEEE 802.11a PHY scheme, while lines that end with " $b$ " at the legend entry were calculated with IEEE 802.11b PHY scheme. It can be observed in Figure 13 that for IEEE 802.11a PHY scheme $\eta$ is as high as 1.38. When the amount of nodes is less than $200, \eta>1$. For IEEE 802.11b PHY

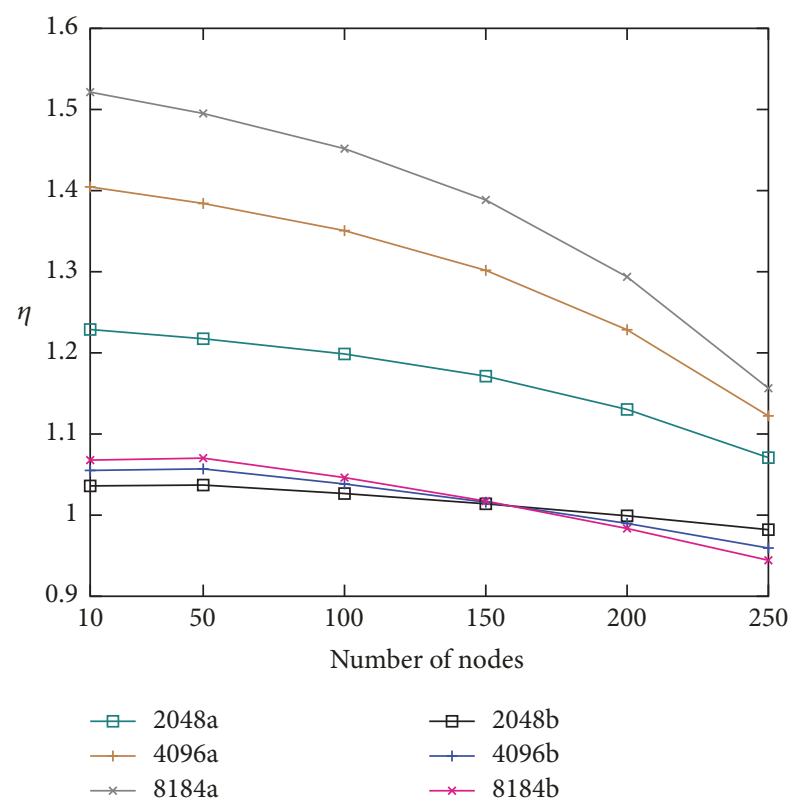

FIgURE 14: Uniform mode throughput comparison when $P_{\mathrm{sz}}(1)=$ 1024.

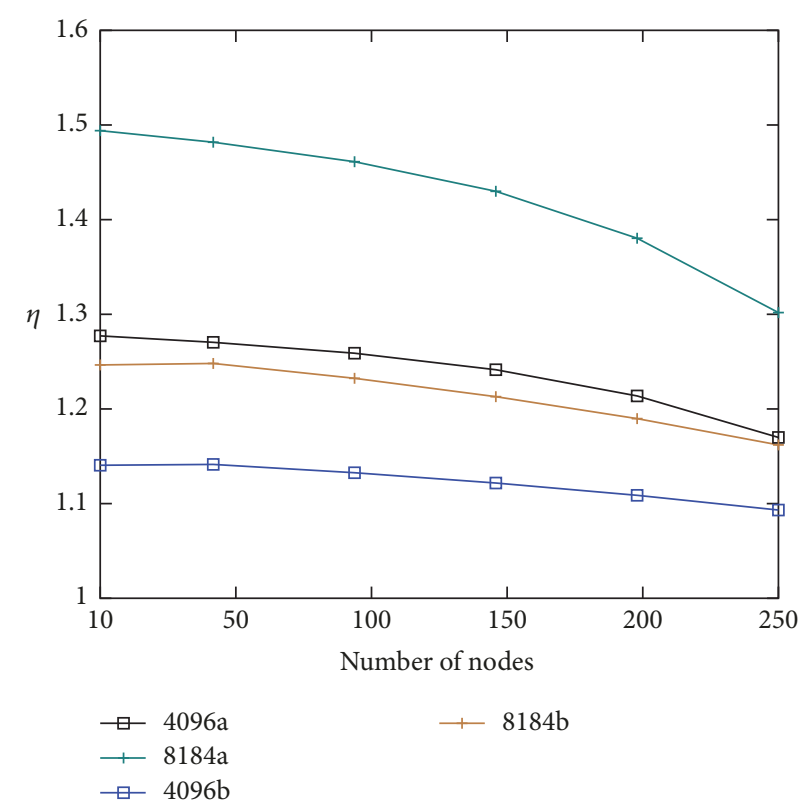

FIGURE 15: Uniform mode throughput comparison when $P_{\mathrm{sz}}(1)=$ 2048.

scheme, the uniform data mode is not efficient when compared with FDT-MAC in these scenarios, since $\eta<1$ for all cases.

Similarly to Figure 13, Figure 14 depicts the results for the scenarios where $P_{\mathrm{sz}}(1)=1024$. In this case, for IEEE 802.11a PHY scheme, uniform data mode achieves higher throughput in all evaluated scenarios, while $\eta$ reaches 1.52 when the amount of nodes is equal to 10. For IEEE 802.11b PHY scheme, $\eta$ is as high as 1.07 and is greater than 1 when the amount of nodes is less than 200 .

Figure 15 presents the results for the scenarios where $P_{\mathrm{sz}}(1)=2048$. In these scenarios, every result of both IEEE 
802.11a and IEEE 802.11b PHY schemes is such that $\eta>1$. $\eta$ reaches at most 1.49 and 1.24 for IEEE 802.11a and IEEE 802.11b PHY schemes, respectively. Also, $\eta$ is always higher than 1.16 and 1.09 for IEEE 802.11a and IEEE 802.11b PHY schemes, respectively. The presented evaluation shows that uniform data mode can improve network performance. However, it is important to find out when its usage may lead to benefit or to losses. In the presented evaluations in Figures 13,14 , and 15 some trends are remarkable:

(1) When the amount of nodes raises, there are losses for uniform data mode performance.

(2) Generally, uniform data mode presented higher values of $\eta$ for larger payload values, since the results when $P_{\mathrm{sz}}(1)=1024$ were much better than the achieved for $P_{\mathrm{sz}}(1)=512$.

(3) When the IEEE 802.11a PHY scheme is considered, $\eta$ is higher than when IEEE 802.11b PHY scheme is considered. However, IEEE 802.11a PHY scheme is more dependent on the amount of nodes, since $\eta$ decays faster.

The first trend occurs since it is more difficult to get a successful transmission when the amount of nodes raises due to the channel contended by the nodes. As uniform data mode performance may split a large DATA packet into two or more shorter packets, it needs to get the channel more times to deliver that DATA than if uniform data mode was not enabled. So, the first trend was expected to occur. In regard to the other trends, for FDT-MAC with uniform data mode enabled, let $\bar{T}^{\prime}$ slot denotes the average time slot duration and $P_{\mathrm{sz}}^{\prime}$ denotes the payload size. So, it can be inferred from (6) that

$$
\begin{aligned}
\eta & =\frac{S^{\prime}}{S}=\frac{\left(p_{s} \cdot p_{\mathrm{tr}} \cdot P_{\mathrm{sz}}^{\prime}\right) / \bar{T}_{\text {slot }}^{\prime}}{\left(p_{s} \cdot p_{\mathrm{tr}} \cdot P_{\mathrm{sz}}\right) / \bar{T}_{\mathrm{slot}}}=\frac{P_{\mathrm{sz}}^{\prime}}{P_{\mathrm{sz}}} \cdot \frac{\bar{T}_{\text {slot }}}{\overline{T_{\text {slot }}^{\prime}}} \\
& =\frac{2 \cdot \min \left(P_{\mathrm{sz}}(1), P_{\mathrm{sz}}(2)\right)}{\left(P_{\mathrm{sz}}(1)+P_{\mathrm{sz}}(2)\right)} \cdot \frac{\bar{T}_{\text {slot }}}{{\overline{T^{\prime}}}_{\text {slot }}} .
\end{aligned}
$$

Equation (12) helps to better understand the second and third trends. As $P_{\mathrm{sz}}(1)$ increases, $2 \cdot\left(\min P_{\mathrm{sz}}(1), P_{\mathrm{sz}}(2)\right)$ grows faster than $\left(P_{\mathrm{sz}}(1)+P_{\mathrm{sz}}(2)\right)$, once $P_{\mathrm{sz}}(1)<P_{\mathrm{sz}}(2)$ holds for the evaluated scenarios. As $P_{\mathrm{sz}}(1)$ is directly proportional to $\eta$, the second trend was expected to occur. Recall from the results presented early in this section (see Section 5.1) that the total transmission time for IEEE 802.11a PHY scheme is shorter than for IEEE 802.11b PHY scheme. As $T_{\text {slot }}$ is also shorter for IEEE 802.11a PHY scheme [7], it can be stated that $\bar{T}_{\text {slot }}^{a}=\bar{T}_{\text {slot }}^{b}-k, k>0$, where $\bar{T}_{\text {slot }}^{a}$ and $\bar{T}_{\text {slot }}^{b}$ denote the average time slot duration for IEEE 802.11a PHY and IEEE 802.11b PHY schemes, respectively. That is, the average time slot for IEEE 802.11a PHY scheme is shorter than for IEEE 802.11b PHY scheme. A similar argument holds for $\bar{T}_{\text {slot' }}$, that is, $\bar{T}_{\text {slot }^{\prime}}^{a}=\bar{T}_{\text {slot }^{\prime}}^{b}-k$. Furthermore, it is trivial that

$$
\frac{x}{y}<\frac{x-k}{y-k}, \quad x>y>k>0 .
$$

Hence,

$$
\frac{\bar{T}_{\text {slot }}^{b}}{\bar{T}_{\text {slot' }^{\prime}}^{b}}<\frac{\bar{T}_{\text {slot }}^{a}}{\bar{T}_{\text {slot' }}^{a}},
$$

once $\bar{T}_{\text {slot }}^{b}>\bar{T}_{\text {slot' }}^{b}$. As $\eta$ is directly proportional to $\bar{T}_{\text {slot }} / \bar{T}_{\text {slot' }}$, $\eta$ is expected to be higher for IEEE 802.11a PHY scheme as pointed in the third trend. As $T_{s}, T_{c}$, and $T_{\text {slot }}$ values are lower for IEEE 802.11a PHY scheme, it makes $\bar{T}_{\text {slot' }}$ to be more affected by the decrease of $p_{\text {tr }}$ that is caused by the rise in the amount of nodes. Thus, $\eta$ decays faster when IEEE 802.11a PHY scheme is considered, as pointed in the third trend.

The presented evaluation about the uniform data mode of FDT-MAC ends the results analysis presented by this work. The referred analysis showed that FDT-MAC had a tremendous positive impact over network performance since it can accomplish a communication faster than traditional schemes, with a negligible probability of error. Our analysis considered both collision-free and heavy traffic scenarios of the network. It is remarkable that FDT-MAC attained relevant improvements in all evaluated scenarios when compared with state-of-the-art full-duplex MAC technique (FD-MAC). Moreover, some of the presented results based on theoretical models were compared with its original values presented by $[8,18,27]$. This comparison showed that our values were very close to the originals, reinforcing the consistency of our results and analysis. Finally, this analysis points that FDT-MAC employment may be a suitable alternative to the existing limitations related to sharing of spectrum resources for full-duplex communications in the $5 \mathrm{G}$ wireless networks context.

\section{Conclusion}

The use of full-duplex antennas has been considered a promising alternative to improve spectrum usage and throughput, especially for $5 \mathrm{G}$ mobile wireless networks. In this context, this work proposed a MAC technique (FDT-MAC) that can accomplish a communication in a fraction of the time wasted by traditional schemes. The throughput improvement measured is as high as $156 \%$ and $412 \%$, when FDT-MAC is compared against FD-MAC and HD-MAC, respectively. Furthermore, uniform data mode is proposed along with FDTMAC in order to allow better spectrum usage and higher throughput. When uniform data mode is properly enabled, FDT-MAC reached a throughput up to $65 \%$ when compared against FD-MAC. As future works, it would be interesting to devise ways to find out a dynamic threshold to enable the uniform data mode, once it may boost dramatically the network performance.

\section{Notations}
$\Delta(G)$ : Maximum degree of graph $G$ (network topology)
$\tau: \quad$ RSSI measure precision $(\mathrm{dB})$


$A(a, b):$ AOA at node $a$ of the signal originated from node $b$

$g(x): \quad$ Antenna gain of node $x$

$G$ : $\quad$ Graph representing the network topology

$h(x)$ : Antenna height

lo $(x): \quad$ System loss of node $x$

$P_{\text {sz }}: \quad$ Data packet payload size in bytes

$P_{f}: \quad$ Prediction error probability

pow $(x)$ : Transmission power of node $x$

$R_{c}: \quad$ Channel transmission data rate (Mbps)

$R_{\text {Max }}: \quad$ Maximum RSS

$R_{\text {Min }}$ : Minimum signal magnitude (in $\mathrm{dB}$ ) detectable by the antenna

$R(a, b)$ : RSS level at node $a$ of the signal originated from node $b$

$\mathrm{sh}_{\text {med }}$ : Average shadowing of the propagation channel

$T_{\text {slot }}: \quad$ Time of duration of a slot time $(\mu \mathrm{s})$

$T_{\mathrm{ACK}}: \quad$ ACK transmission time $(\mu \mathrm{s})$

$T_{B}: \quad$ Expected backoff time $(\mu \mathrm{s})$

$T_{\mathrm{CT}}: \quad$ CTS transmission time $(\mu \mathrm{s})$

$T_{\text {DATA }}: \quad$ Data packet transmission time $(\mu \mathrm{s})$

$T_{\mathrm{DF}}: \quad$ DCF interframe space time of IEEE 802.11 standard $(\mu \mathrm{s})$

$T_{p}: \quad$ Pulse/tone transmission time $(\mu \mathrm{s})$

$T_{\mathrm{RT}}: \quad$ RTS transmission time $(\mu \mathrm{s})$

$T_{\mathrm{SF}}: \quad$ Short interframe space time of IEEE 802.11 standard $(\mu \mathrm{s})$

$w$ : Ratio between channel propagation speed and speed of light.

\section{Conflicts of Interest}

The authors declare that there are no conflicts of interest regarding the publication of this article.

\section{Acknowledgments}

This work was partially supported by DPP/UnB.

\section{References}

[1] H. Wang, S. Chen, H. Xu, M. Ai, and Y. Shi, "SoftNet: a software defined decentralized mobile network architecture toward 5G," IEEE Network, vol. 29, no. 2, pp. 16-22, 2015.

[2] R. Tang, J. Zhao, H. Qu, and Z. Zhang, "Energy-efficient resource allocation for $5 \mathrm{G}$ full-duplex enabled device-to-device communication," in Proceedings of the 2016 IEEE Globecom Workshops (GC Wkshps), pp. 1-7, IEEE, Washington, DC, USA, December 2016.

[3] F. Boccardi, R. W. Heath, A. Lozano, T. L. Marzetta, and P. Popovski, "Five disruptive technology directions for 5G," IEEE Communications Magazine, vol. 52, no. 2, pp. 74-80, 2014.

[4] C.-X. Wang, F. Haider, X. Gao et al., "Cellular architecture and key technologies for $5 \mathrm{G}$ wireless communication networks," IEEE Communications Magazine, vol. 52, no. 2, pp. 122-130, 2014.

[5] X. Zhang, W. Cheng, and H. Zhang, "Full-duplex transmission in phy and mac layers for $5 \mathrm{G}$ mobile wireless networks," IEEE
Wireless Communications Magazine, vol. 22, no. 5, pp. 112-121, 2015.

[6] Z. Zhang, K. Long, A. V. Vasilakos, and L. Hanzo, "Full-duplex wireless communications: challenges, solutions, and future research directions," Proceedings of the IEEE, vol. 104, no. 7, pp. 1369-1409, 2016.

[7] IEEE, "IEEE Standard for information technology-telecommunications and information exchange between systemslocal and metropolitan area networks - specific requirements - part 11: Wireless lan medium access control (MAC) and physical layer (PHY) specifications," Institute of Electrical and Electronics Engineers, IEEE Standard 802.11, December 2007.

[8] J. Jun, P. Peddabachagari, and M. Sichitiu, "Theoretical maximum throughput of IEEE 802.11 and its applications," in Proceedings of the 2nd IEEE International Symposium on Network Computing and Applications, NCA 2003, pp. 249-256, Cambridge, MA, USA, April 2003.

[9] L. D. E. M. Guimarães, J. L. Bordim, and K. Nakano, "Using pulse/tone signals as an alternative to boost channel reservation on directional communications," IEICE Transactions on Fundamentals of Electronics, Communications and Computer Sciences, vol. E98A, no. 8, pp. 1647-1656, 2015.

[10] J. Choi II, M. Jain, K. Srinivasan, P. Levis, and S. Katti, "Achieving single channel, full duplex wireless communication," in Proceedings of the 16th Annual Conference on Mobile Computing and Networking (MobiCom '10), pp. 1-12, ACM, September 2010.

[11] R. Choudhury and N. Vaidya, "Deafness: a MAC problem in ad hoc networks when using directional antennas," in Proceedings of the 12th IEEE International Conference on Network Protocols, ICNP, pp. 283-292, IEEE, Berlin, Germany, 2004.

[12] K.-P. Shih, W.-H. Liao, H.-C. Chen, and C.-M. Chou, "On avoiding RTS collisions for IEEE 802.11-based wireless ad hoc networks," Computer Communications, vol. 32, no. 1, pp. 69-77, 2009.

[13] M. Malajner, P. Planinsic, and D. Gleich, "Angle of arrival estimation using RSSI and omnidirectional rotatable antennas," IEEE Sensors Journal, vol. 12, no. 6, pp. 1950-1957, 2012.

[14] G. Giorgetti, S. Maddio, A. Cidronali, S. Gupta, and G. Manes, "Switched beam antenna design principles for angle of arrival estimation," in Proceedings of the in Proceedings fo the European Wireless Technology Conference, pp. 5-8, IEEE, Rome, Italy, 2009.

[15] J. Lee, D. Kim, C. Toh, T. Kwon, and Y. Choi, "A Table-driven AOA Estimation Algorithm for Switched-beam Antennas in Wireless Networks," in Proceedings of the 11th European Wireless Conference, pp. 1-6, VDE, Nicosia, Cyprus, April 2005.

[16] A. A. Sani, L. Zhong, and A. Sabharwal, "Directional antenna diversity for mobile devices: Characterizations and solutions," in Proceedings of the 16th Annual Conference on Mobile Computing and Networking, MobiCom 2010, pp. 221-232, Chicago, Illinois, USA, September 2010.

[17] J. Liberti and T. Rappaport, Smart Antennas for Wireless Communications, Prentice Hall PTR, Upper Saddle River, NJ, USA, 1999.

[18] W. Cheng, X. Zhang, and H. Zhang, "RTS/FCTS mechanism based full-duplex MAC protocol for wireless networks," in Proceedings of the 2013 IEEE Globecom Workshops (GC Wkshps), pp. 5017-5022, IEEE, Atlanta, GA, USA, 2013.

[19] S. Basagni, M. Conti, S. Giordano, and I. Stojmenovic, Mobile Ad Hoc Networking, John Wiley \& Sons, Inc., Hoboken, NJ, USA, 2004. 
[20] G. Mao, B. D. O. Anderson, and B. Fidan, "Path loss exponent estimation for wireless sensor network localization," Computer Networks, vol. 51, no. 10, pp. 2467-2483, 2007.

[21] P. Mohapatra and S. V. Krishnamurthy, Ad Hoc Networks: Technologies And Protocols, Springer, New York, NY, USA, 2005.

[22] T. S. Nunnikhoven, "A birthday problem solution for nonuniform birth frequencies," The American Statistician, vol. 46, no. 4, pp. 270-274, 1992.

[23] T. Sakano, Z. Fadlullah, T. Ngo et al., "Disaster-resilient networking: a new vision based on movable and deployable resource units," IEEE Network, vol. 27, no. 4, pp. 40-46, 2013.

[24] W. Liu, H. Nishiyama, N. Kato, Y. Shimizu, and T. Kumagai, "A novel gateway selection method to maximize the system throughput of Wireless Mesh Network deployed in disaster areas," in Proceedings of the 2012 IEEE 23rd International Symposium on Personal, Indoor and Mobile Radio Communications, PIMRC 2012, pp. 771-776, Australia, September 2012.

[25] S. Ullah, M. Chen, and K. S. Kwak, "Throughput and delay analysis of IEEE 802.15.6-based CSMA/CA protocol," Journal of Medical Systems, vol. 36, no. 6, pp. 3875-3891, 2012.

[26] E. H. Ong, J. Kneckt, O. Alanen, Z. Chang, T. Huovinen, and T. Nihtila, "IEEE 802.11ac: enhancements for very high throughput WLANs," in Proceedings of the 22nd International Symposium on Personal, Indoor and Mobile Radio Communications (PIMRC '11), pp. 849-853, IEEE, September 2011.

[27] G. Bianchi, "Performance analysis of the IEEE 802.11 distributed coordination function," IEEE Journal on Selected Areas in Communications, vol. 18, no. 3, pp. 535-547, 2000.

[28] I. Tinnirello, G. Bianchi, and Y. Xiao, "Refinements on IEEE 802.11 distributed coordination function modeling approaches," IEEE Transactions on Vehicular Technology, vol. 59, no. 3, pp. 1055-1067, 2010.

[29] E. Ziouva and T. Antonakopoulos, "CSMA/CA performance under high traffic conditions: throughput and delay analysis," Computer Communications, vol. 25, no. 3, pp. 313-321, 2002.

[30] M. Ergen and P. Varaiya, "Throughput analysis and admission control for IEEE 802.11a," Mobile Networks and Applications, vol. 10, no. 5, pp. 705-716, 2005.

[31] S. Sagari, S. Baysting, D. Saha, I. Seskar, W. Trappe, and D. Raychaudhuri, "Coordinated dynamic spectrum management of LTE-U and Wi-Fi networks," in Proceedings of the IEEE International Symposium on Dynamic Spectrum Access Networks, DySPAN 2015, pp. 209-220, Sweden, October 2015.

[32] Y. Yao, L. Rao, and X. Liu, "Performance and reliability analysis of IEEE 802.11p safety communication in a highway environment," IEEE Transactions on Vehicular Technology, vol. 62, no. 9, pp. 4198-4212, 2013.

[33] P. Nayak, M. Garetto, and E. W. Knightly, "Multi-user downlink with single-user uplink can starve TCP," in Proceedings of the INFOCOM 2017-IEEE Conference on Computer Communications, pp. 1-9, IEEE, Atlanta, GA, USA, May 2017. 


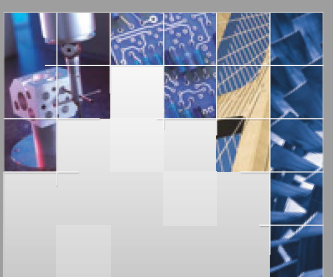

\section{Enfincering}
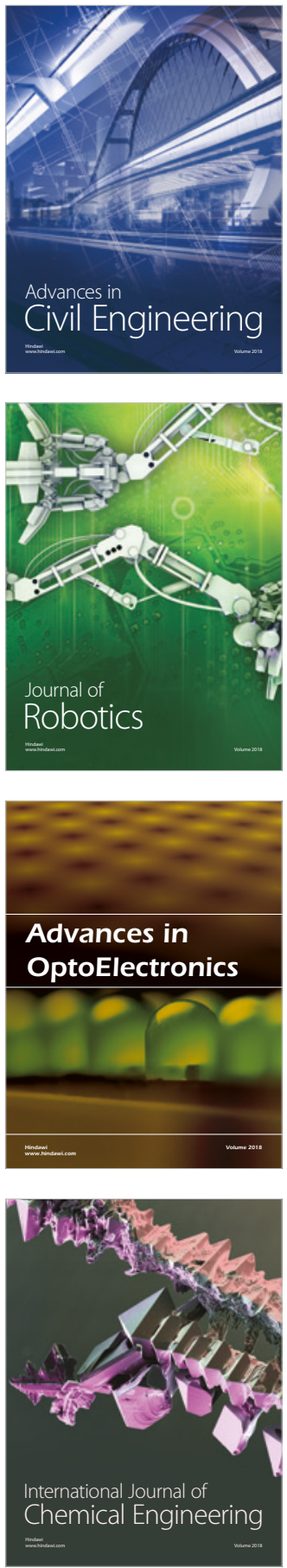

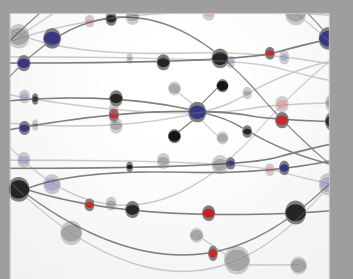

\section{Rotating \\ Machinery}

The Scientific World Journal

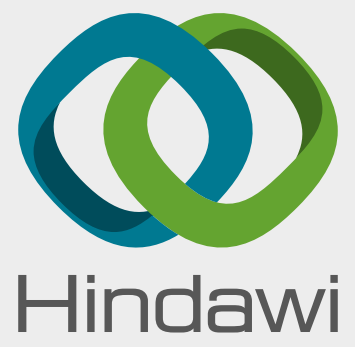

Submit your manuscripts at

www.hindawi.com
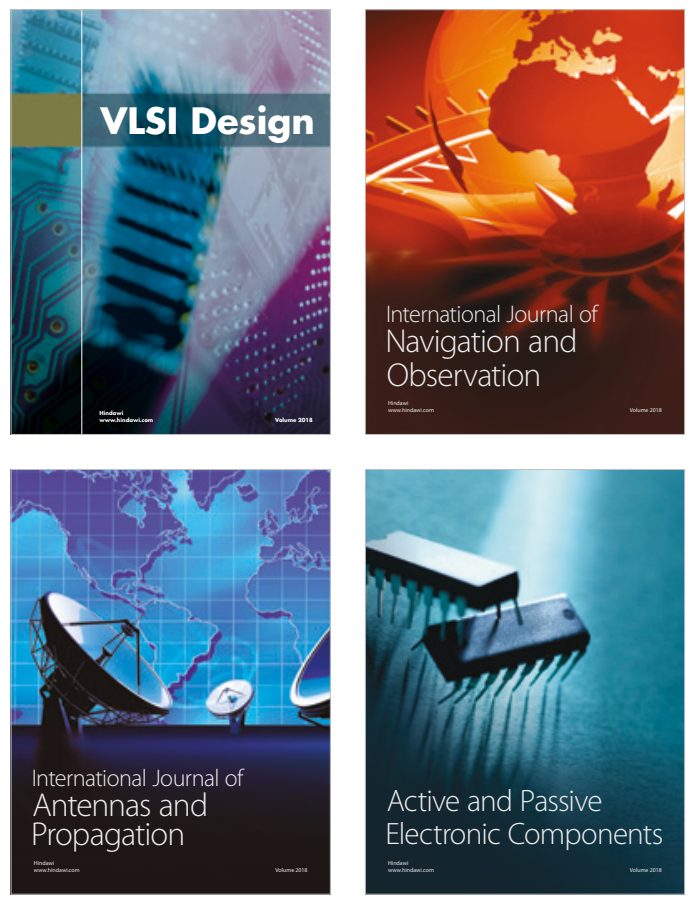
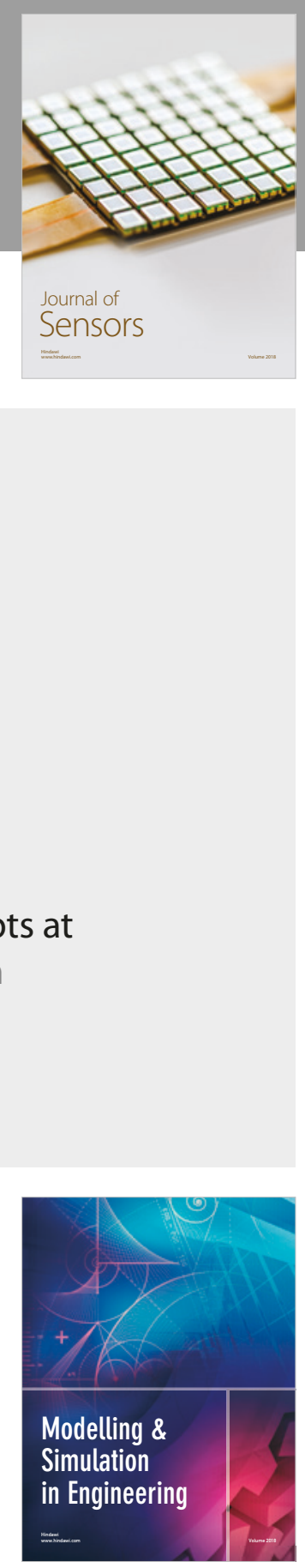

\section{Advances \\ Multimedia}
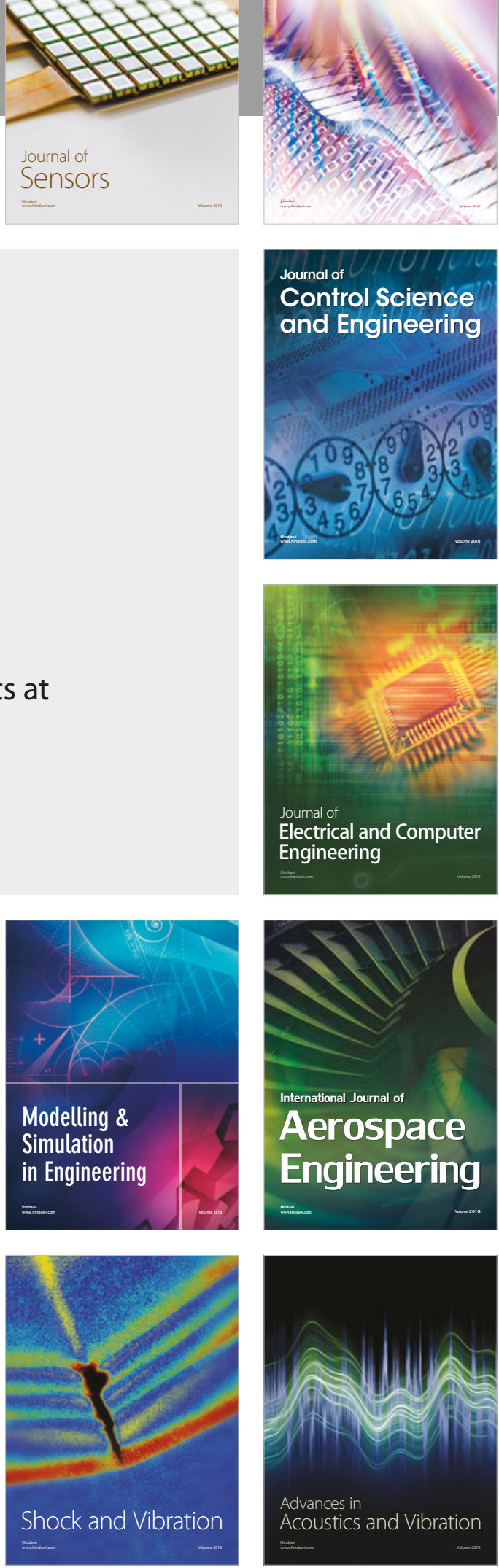